\title{
THERMAL RADIATION AND JOULE HEATING EFFECTS ON A MAGNETOHYDRODYNAMIC CASSON NANOFLUID FLOW IN THE PRESENCE OF CHEMICAL REACTION THROUGH A NON-LINEAR INCLINED POROUS STRETCHING SHEET
}

\section{B. Shankar Goud ${ }^{1}$, Y. Dharmendar Reddy ${ }^{2}$, V. Srinivasa Rao ${ }^{3}$, Zafar Hayat Khan ${ }^{4}$}

${ }^{1}$ Department of Mathematics, JNTUH College of Engineering, Hyderabad, Telangana State, India - 500085. Email: bsgoud.mtech@gmail.com

${ }^{2}$ Department of Mathematics, Anurag University, Hyderabad, Telangana State, India- 500088. Email: dharmayanala@gmail.com

${ }^{3}$ Department of Mathematics, Anurag University, Hyderabad, Telangana State, India- 500088. Email: uhita@yahoo.com

${ }^{4}$ State Key Laboratory of Hydraulics and Mountain River Engineering, College of Water Resource and Hydropower, Sichuan University, Chengdu 610065, P. R. China. Email: zafarhayyatkhan@gmail.com

\begin{abstract}
:
The present study explores the thermal and Joule heating effect of Casson nanofluid flow with chemical reaction over an inclined porous stretching surface. The results of heat source/sink, viscous dissipation, and suction are regarded. The new physical governing equations of partial differential flow equations are converted into nonlinear ordinary differential equations and are numerically resolved employing the implicit finite difference technique. The influence on velocity, temperature, and concentration fields of many flow variables are addressed. It is found that the angle of inclination parameter leads to enhance in the boundary layer thickness and also identified that the Joule heating parameter increases the thermal boundary layer thickness which helps in holding system temperature of the fluid flow. The numerical and graphical findings are defined for the numerous related attentiveness flow parameters. The empirical data reported are compared with the published outcomes. In addition, the skin friction coefficient, Nusselt and Sherwood numbers were shown in tabular form and significant changes were observed with the influence of various parameters.
\end{abstract}

Keywords: Thermal radiation, MHD, Casson nanofluid, Joule heating, suction, chemical reaction, Keller Box method.

\section{NOMENCLATURE}

$\begin{array}{cl}u, v & \text { factors of velocity in } \mathrm{x} \text { and } \mathrm{y} \text {-direction } \\ \eta & \text { Dimensionless variable } \\ \alpha^{*} & \text { thermal diffusivity } \\ a & \text { positive constant } \\ Q_{0} & \text { volumetric heat generation/absorption } \\ \beta_{T} & \text { coefficient of thermal expansion } \\ v & \text { kinematic viscosity } \\ \mu & \text { dynamic viscosity of a fluid } \\ C & \text { fluid concentration } \\ T & \text { fluid temperature } \\ S c=\frac{v}{D_{B}} & \text { Schmidt number } \\ T_{w} & \text { wall temperature }\end{array}$

\begin{tabular}{cl}
$E c=\frac{U_{w}{ }^{2}}{\left(T_{w}-T_{\infty}\right) c_{p}}$ & Eckert number \\
$G c=\frac{g \beta_{C}\left(C_{w}-C_{\infty}\right)}{a^{2} x^{(2 n-1)}}$ & local modified Grashoff number \\
$H a=\frac{2 \sigma B_{0}{ }^{2}}{a \rho_{f}(n+1)}$ & Hartmann Number \\
$T_{\infty}$ & ambient temperature \\
$D_{T}$ & Thermophoresis diffusion \\
$N b=\frac{(\rho c)_{p} D_{B}\left(C_{w}-C_{\infty}\right)}{v(\rho c)_{f}}$ & Brownian motion parameter \\
$\gamma=\frac{2 x k_{0}}{(n+1) U_{w}}$ & Chemical reaction parameter \\
$G r=\frac{g \beta_{T}\left(T_{w}-T_{\infty}\right)}{a^{2} x^{(2 n-1)}}$ & local Grashoff number \\
$\boldsymbol{J}=\frac{C_{w}}{a \rho_{f}\left(T_{w}-T_{\infty}\right) C_{p}(n+1)}$ & nanoparticle concentration \\
$\boldsymbol{Q}=\frac{2 x \boldsymbol{Q}_{0}}{(\rho c)_{f}(n+1) U_{w}}$ & Heat source/sink parameter \\
\hline All rights reserved. & Received on: Oct., 2020
\end{tabular}

1813-8535 (Print), 2070-8998 (Online) @ 2020 ANAME Publication. All rights reserved.

Received on: Oct., 2020 


\begin{tabular}{|c|c|c|c|}
\hline $\begin{array}{c}\alpha \\
B(x)\end{array}$ & $\begin{array}{l}\text { an inclination parameter } \\
\text { non-uniform magnetic field }\end{array}$ & $S=-\frac{\operatorname{Re}_{x}}{\sqrt{\frac{a v(n+1)}{2} x} x^{\left(\frac{n-1}{2}\right)}}$, & $\begin{array}{l}\text { local Reynolds number } \\
\text { Suction parameter }\end{array}$ \\
\hline$\sigma^{*}$ & Stefan-Boltzman constant & $\rho_{f}$ & base fluid density \\
\hline$g$ & acceleration due to gravity & $B_{0}$ & uniform magnetic field \\
\hline$C f_{x}$ & skin friction coefficient & $\psi$ & Stream function \\
\hline$D_{B}$ & Brownian diffusion coefficient & $R=\frac{4 \sigma T_{\infty}^{3}}{\kappa^{*} k}$ & Radiation parameter \\
\hline $\begin{array}{c}S h_{x} \\
U_{w}=a x^{\frac{n-1}{2}}\end{array}$ & $\begin{array}{l}\text { local Sherwood number } \\
\text { stretching velocity }\end{array}$ & $\kappa^{\star} K$ & electrical conductivity \\
\hline $\operatorname{Pr}=\frac{v}{\alpha}$ & Prandtl number & $\beta$ & Casson fluid parameter \\
\hline$N u_{x}$ & local Nusselt number & $C_{\infty}$ & $\begin{array}{l}\text { ambient value of nanoparticle } \\
\text { fraction }\end{array}$ \\
\hline$n$ & nonlinear stretching parameter & $\tau_{w}$ & Wall shear stress \\
\hline$c_{p}$ & specific heat capacity & $\beta_{C}$ & $\begin{array}{l}\text { Concentration expansion } \\
\text { coefficient }\end{array}$ \\
\hline$v_{w}$ & velocity of suction & $q_{w}$ & heat flux at the wall \\
\hline$k^{*}$ & mean absorption coefficient & $w$ & condition at the surface \\
\hline$q_{m}$ & the mass flux & $q_{r}$ & the radiative heat flux \\
\hline$B_{0}$ & Constant & $x, y$ & space coordinates \\
\hline$k_{0}$ & chemical reaction coefficient & $(\rho c)_{f}$ & heat capacity of the fluid \\
\hline$k$ & Nanofluid thermal conductivity & $N t=\frac{(\rho c)_{p} D_{T}\left(T_{w}-T_{\infty}\right)}{\nu(\rho c)_{f} T_{\infty}}$ & Thermophoresis parameter \\
\hline$(\rho c)_{p}$ & effective heat capacity of a nanoparticle & $\infty$ & condition at the free stream \\
\hline
\end{tabular}

\section{Introduction}

Many Researchers are involved in addressing the fluid flow issues across stretching surfaces. Such flows are ideal for applications in the paper, plastic, fiber, silicone, and metal sheets industries (Yasin et a., 2016, Nayak et al., 2016, Ashraf and Bashir, 2011, Adhikari and Sanyal, 2013, sui et al., 2015). Magnetohydrodynamics, a significant area of study, and plays a key role in engineering and science which include the electrically induced physiological fluid movement under the magnetic field results. Maxwell's equation described the simulation of these fluids. The induction of electrical impulses into flowing fluids induces transverse magnetic fields. The collaboration of these induced currents with a magnetic field is known as the Lorentz force as a sort of resistive energy. Different magnetic field applications are growing in energy equipment are studied by Babu et al. (2014), smart aerospace production by Shima et al. (2009) and industrial processing devices by Ferdows et al. (20014).

The flow studies of the porous medium have become very prominent due to major functional applications in various sciences and engineering sectors, in which few are polymer extrusion, heat swapping, geophysical, geothermal, etc., as well as in soil water purification, petroleum tanks, kernel storage systems, etc. The thermal and mass transport of the MHD flow has become a subject of work in current days because of influential applications in engineering and manufacturing. Particularly in the production of magnetic materials, geophysics, and cooling rate control, etc. For this purpose, Makinde and Aziz (2010) deliberate the mixed convection MHD boundary layer flow combined with a vertical porous plate. Pekmen et al. (2019) considered with the magnetic effect, Arifuzzaman et al. (2018) investigated the behavior of radiation and homogeneous-heterogeneous effects. Raptis et al. (1982) presented the free convective MHD flow with constant thermal flux on an infinite vertical plate. For business and science, the primary source of radiation is the public welfare, and lastly, the veterinary. The impact of radiation on fluid flows is worth analyzing, for instance, nuclear protection, geothermal plants, heat internment, etc. Chemical reaction erudition is used in immense technical and technological sectors, including the chemical industry, material manufacturing, food manufacturing and glass development. For 
example, in recent studies Pilani and Kim (2012) examined the heat transfer characteristics of natural convection past a vertical cone under the combined effects of magnetic field and thermal radiation with subjected to a variable surface heat flux. A steady two-dimensional MHD free convection of heat transfer in flow past a semi-infinite flat plate in transverse magnetic field with heat flux has been examined by Geetha et.al. (2014), Khana et al. (2018) discussed the unsteady flow of non-Newtonian fluid with the properties of heat source/sink in the presence of thermal radiation moving through a binary mixture embedded in a porous medium. Shankar and Rajashekar (2018) studied the influence of MHD heat transfer fluid flow of a micropolar fluid over a stretching surface, Mohamed and Abo-Dahab (2009) analyzed the effects of chemical reaction and thermal radiation on hydromagnetic free convection heat and mass transfer micropolar fluid over a porous medium bounded by a semi-infinite vertical porous plate with heat generation, Nandeppanavar et al. (2011) are examined the flow and heat transfer characteristics for MHD viscoelastic boundary layer flow over an impermeable stretching sheet with space and temperature dependent internal heat generation/absorption, thermal radiation due to frictional heating.

The word nanofluid was first used by Choi (1995) to refer to fluids with suspended nanoparticles. In recent years, work on heat transfer and boundary layer flow in nanofluids has earned expanded aid in various industrial applications owing to its growing relevance. Daniel et al. (2018), Akbar (2013), Makinde and Aziz (2011) studied the different aspects on magnetohydrodynamics (MHD) flow of nanofluid towards a linear and nonlinear stretching surface in the presence of thermal radiation, viscous dissipation, Joule heating, and chemical reaction. The fluid disobeys Newton's law of viscosity is abbreviated as non-Newtonian fluids. Casson fluid is the Non-Newtonian fluid. Few daily lives Casson examples include blood, honey, fruit juices, etc. Casson's fluid also has significant uses in research, electronics and medicine, oil recovery systems, and cooling computer tools that dry rigid structures. The Casson fluid is more capable than the Newtonian fluid in the process of thermal transport. Non-Newtonian fluid properties, including Casson fluid and due to their broad uses in several fields manufacturing. Casson fluid is a solvent that dilutes the shear and at zero. This would have infinite viscosity and vice versa shear values. Some investigations are made by many scientists and scholars were inspired by the Mustafa et al. (2011), Nandy et al. (2013), Bhattacharyya (2013), Mukhopadhyay et al.(2013), Hari Singh Nayak et al. (2020), Hayat et al. (2012), Nadeem et al. (2014) considered MHD stagnation-point flow and Casson fluid flow with varying impacts, and some of the authors are studied the impact of radiation, viscous dissipation, heat source/sink on magnetohydrodynamic nanofluid heat transfer boundary layer flow over a stretching sheet with slip conditions and variable suction (Shankar Goud et al, (2020), Dharmendar Reddy et al., 2016, Shankar Goud, 2020, Dharmendar Reddy et al., 2017, Bal Reddy et al. 2018). Khan et al. (2014) and Akhbar et al. (2015) discussed the impact of MHD boundary layer flow of a nanofluid containing gyrotactic microorganisms past a vertical plate with Navier slip and MHD dissipative flow and heat metachronal wave propulsion of velocity slip effects, Rizwan-Ul-Haq et al. (2014) analyzed the MHD convective heat transfer effects on Casson nanofluid flow over a shrinking sheet, Nayak et al. (2017) studied the thermal radiation impact on three-dimensional free convective flow of nanofluid over a linear stretching sheet. Sharma and Saboo (2017) discussed the heat and mass transfer with viscous dissipation in horizontal channel partially occupied by porous medium in the presence of oscillatory suction. Effect of radiation and chemical reaction on MHD flow past a vertical plate with variable temperature and mass diffusion was studied by Rajput and Gaurav Kumar (2019). Bala Anki Reddy (2016) studied the effect of MHD boundary layer slip flow of a Casson fluid over an exponentially stretching surface in the presence of thermal radiation and chemical reaction. Numerical analysis of Heat and Mass transfer along a stretching wedge surface are analyzed by Ali et al. (2017). Further, Banshiwal and Goyal (2018) have examined the influence of MHD non-darcian flow due to horizontal stretching sheet embedded in a porous medium with thermal stratification effects.

The objective of this study is mainly concentrated on the influence of source /sink, Joule heating and thermal radiation on MHD free convection flow of a Casson Nanofluid over a inclined non-linear stretching sheet in presence of chemical reaction. The transformed nonlinear ordinary differential equations are solved numerically by using Keller-Box method. The effect of various physical flow parameters on velocity, temperature and concentration distributions are presented graphically. In the present study, the obtained results are compared with previously published work and found to be in a good agreement. This inclined non-linear porous stretching sheet has extreme coverage in mechanical, civil, aeronautical structure and marine and designs. 


\section{Mathematical Formulation}

This article intensifies electrically conducting incompressible and viscous steady state two dimensional MHD Casson nanofluid mixed convection flow through an inclined nonlinear permeable stretching surface with heat generation/absorption, Joule heating, suction, viscous dissipation, chemical reaction, and thermal radiation placed at $y=0$. The velocity $U_{w}=a x^{\frac{n-1}{2}}(a>0)$ of the sheet changes with the direction of $x-$ the axis and along $y$-direction with the velocity of $v=v_{w}$. In addition, the current flow scope is positive $(y>0)$. Furthermore, this plate is often implemented with the $B(x)=B_{0} x^{\frac{n-1}{2}}$ equation by a magnetic field, which is in the path of $y$-axis. The fluid conducted electrically is induced by the same magnetic field. It should be indicated. The induced magnetic field is close to zero if the Reynolds number is extremely small. Along with this, considered chemical reaction and heat source to the flow. In order to extend the wall such that origin is fixed, while two identical and reverse forces are added along the x-axis direction. In this, $T_{w}$ and $C_{w}$ are considered to be the thermal temperature and concentration of nanoparticle and, as $y \rightarrow \infty$, temperature and nanoparticle volume fraction ambient values are $T_{\infty}$ and $C_{\infty}$. The rheological equation of mood for the as incompressible and isotropic flow of Casson fluid is stated as:

$$
\tau_{i j}= \begin{cases}2\left(\mu_{B}+\frac{p_{y}}{\sqrt{2 \pi}}\right) e_{i j}, & \pi>\pi_{c} \\ 2\left(\mu_{B}+\frac{p_{y}}{\sqrt{2 \pi_{c}}}\right) e_{i j}, & \pi_{c}>\pi\end{cases}
$$

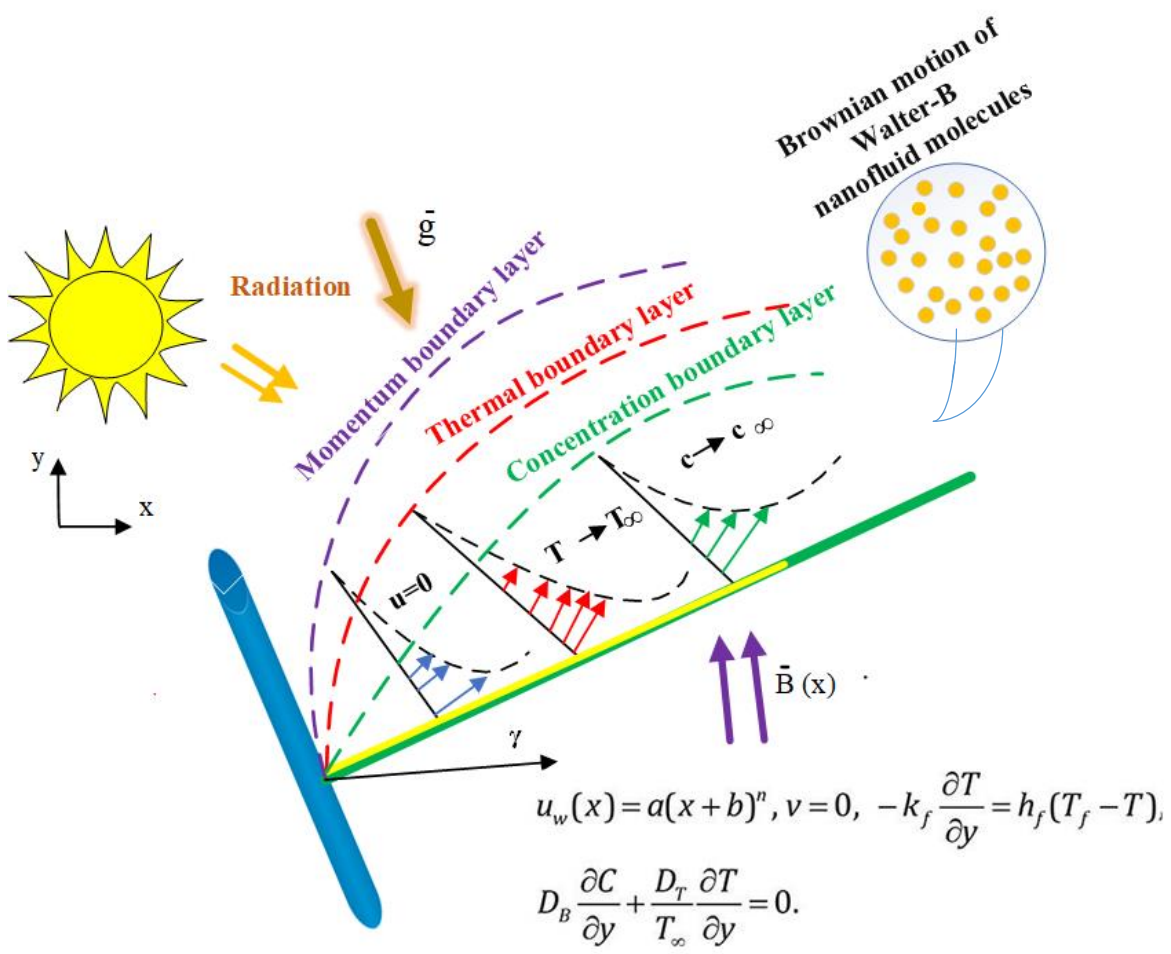

Fig.1: Physical Configuration and Coordinate System

here the fluid yield stress is $p_{y}, \pi_{c}$ is the critical value of this product based on the non-Newtonian model, $\mu_{B}$ is the dynamic non-Newtonian fluid viscosity, $\pi$ is the product of the element of deformation rate with 
itself, and $e_{i j}$ is the $(i, j)^{t h}$ element of the deformation rate.

The following are the set of differential equations, which governs the fluid flow: (Refs. Khan et al. (2014))

$$
\begin{aligned}
& \frac{\partial u}{\partial x}+\frac{\partial v}{\partial y}=0 \\
& u \frac{\partial u}{\partial x}+v \frac{\partial v}{\partial y}=v\left(1+\frac{1}{\beta}\right) \frac{\partial^{2} u}{\partial y^{2}}-\frac{\sigma B^{2}(x)}{\rho_{f}} u+g \beta_{T}\left(T-T_{\infty}\right)+g \beta_{c}\left(C-C_{\infty}\right) \cos \alpha \\
& u \frac{\partial T}{\partial x}+v \frac{\partial T}{\partial y}=\alpha^{*} \frac{\partial^{2} T}{\partial y^{2}}+\frac{(\rho c)_{p}}{(\rho c)_{f}}\left(\frac{D_{T}}{T_{\infty}}\left(\frac{\partial T}{\partial y}\right)^{2}+D_{B} \frac{\partial C}{\partial y} \frac{\partial T}{\partial y}\right)+\frac{v}{c_{p}}\left(1+\frac{1}{\beta}\right)\left(\frac{\partial u}{\partial y}\right)^{2} \\
& +\frac{\sigma B^{2}(x)}{(\rho c)_{f}} u^{2}-\frac{1}{(\rho c)_{f}} \frac{\partial q_{r}}{\partial y}+\frac{1}{(\rho c)_{f}} \frac{Q_{0}}{\left(T-T_{\infty}\right)}, \\
& u \frac{\partial C}{\partial x}+v \frac{\partial C}{\partial y}=D_{B}\left(\frac{\partial^{2} C}{\partial y^{2}}\right)+\frac{D_{T}}{T_{\infty}}\left(\frac{\partial^{2} T}{\partial y^{2}}\right)-k_{0}\left(C-C_{\infty}\right)
\end{aligned}
$$

Roseland approximation of radiative heat flux is yielded by $q_{r}=-\frac{4 \sigma^{*}}{3 k^{*}} \frac{\partial T^{4}}{\partial y}$,

The Taylor series equation for $T^{4}$ as $T_{\infty}$ is the following if a minor temperature variation in flow is assumed:

$T^{4} \approx 4 T T_{\infty}^{3}-3 T_{\infty}^{4}$

Now, substitute Eqs. $(6,7)$ in Eq. (4) then it becomes:

$$
\begin{aligned}
& u \frac{\partial T}{\partial x}+v \frac{\partial T}{\partial y}=\alpha^{*} \frac{\partial^{2} T}{\partial y^{2}}+\frac{(\rho c)_{p}}{(\rho c)_{f}}\left(\frac{D_{T}}{T_{\infty}}\left(\frac{\partial T}{\partial y}\right)^{2}+D_{B} \frac{\partial C}{\partial y} \frac{\partial T}{\partial y}\right)+\frac{v}{c_{p}}\left(\frac{1+\beta}{\cdot \beta}\right)\left(\frac{\partial u}{\partial y}\right)^{2} \\
& +\frac{\sigma B^{2}(x)}{(\rho c)_{f}} u^{2}+\frac{1}{(\rho c)_{f}} \frac{16 \sigma^{*}}{3 k^{*}} \frac{\partial}{\partial y}\left(T^{3} \frac{\partial T}{\partial y}\right)+\frac{1}{(\rho c)_{f}} \frac{Q_{0}}{\left(T-T_{\infty}\right)},
\end{aligned}
$$

Subject to the corresponding conditions are:

$$
\begin{aligned}
& u=U_{w}=a x^{\frac{n-1}{2}}, T=T_{w}, v=v_{w}, C=C_{w} \quad \text { at } \quad y=0, \\
& v \rightarrow 0, T \rightarrow T_{\infty}, u \rightarrow 0, C \rightarrow C_{\infty} \quad \text { as } \quad y \rightarrow \infty
\end{aligned}
$$

It is supposed that $B(x)=B_{0} x^{\left(\frac{n-1}{2}\right)}$ is called the variable magnetic field. To transform the PDE's into ODE's, with the help of the following similarity transformations:

$$
\begin{aligned}
& \eta=y \sqrt{\frac{(n+1) a}{2 v}} x^{\left(\frac{n-1}{2}\right)}, \psi=\sqrt{\frac{2 a v}{(n+1)}} x^{\left(\frac{n-1}{2}\right)} f(\eta), v=-\sqrt{\frac{a v(n+1)}{2}} x^{\left(\frac{n-1}{2}\right)}\left(f(\eta)+\frac{n-1}{n+1} \eta f^{\prime}(\eta)\right), \\
& u=a x^{n} f^{\prime}(\eta), \phi(\eta)=\frac{C-C_{\infty}}{C_{w}-C_{\infty}}, \theta(\eta)=\frac{T-T_{\infty}}{T_{w}-T_{\infty}}
\end{aligned}
$$

Here the local similarity variable is $\eta, f(\eta)$ is the dimensionless stream function, $\theta(\eta), \phi(\eta)$ are the nondimensional temperature and concentration of the nanofluid, Normal description of the stream function $\psi$ specified as $u=\frac{\partial \psi}{\partial y}, v=-\frac{\partial \psi}{\partial x}$. 
Here, take over $v_{w}=-\sqrt{\frac{a(n+1) v}{2}} x\left(\frac{n-1}{2}\right) S$, (the suction parameter is $S$ ).

After replacing Eq. (10) into the Eq.(2), (3), (5), (8) and boundary conditions (9) the ODE's are finally obtained as follows:

$$
\begin{aligned}
& \left(1+\frac{1}{\beta}\right) f^{\prime \prime \prime}+f f^{\prime \prime}-\frac{2}{n+1}\left(n \cdot f^{\prime 2}-G r \cdot \theta+G c \cdot \phi \cdot \cos \alpha\right)-H a \cdot f^{\prime}=0, \\
& \left(1+\frac{4 R}{3}\right) \theta^{\prime \prime}+\operatorname{Pr}\left(f \theta^{\prime}+N b \theta^{\prime} \phi^{\prime}+N t \theta^{\prime 2}+\left(1+\frac{1}{\beta}\right) E c f^{\prime \prime 2}+Q \theta+J f^{\prime 2}\right)=0, \\
& \phi^{\prime \prime}+S c f \phi^{\prime}+\frac{N t}{N b} \theta^{\prime \prime}-S c \gamma \phi=0 .
\end{aligned}
$$

The boundary conditions are

$$
\begin{gathered}
f=S, f^{\prime}=1, \theta=1, \phi=1 \quad \text { at } \quad \eta=0, \\
f^{\prime}=0, \quad \theta=0, \quad \phi=0 \quad \text { as } \quad \eta \rightarrow \infty .
\end{gathered}
$$

where prime (') represents the diff. w. r. to $\eta$.

The physical parameters and non-dimensional numbers are described as follows:

$$
\begin{aligned}
& C f_{x}=\frac{\tau_{w}}{\rho U_{w}{ }^{2}} \text {, where wall shear stress, is } \tau_{w}=\mu_{B}\left(1+\frac{1}{\beta}\right)\left(\frac{\partial u}{\partial y}\right)_{y=0} \\
& N u_{x}=x q_{w} / k\left(T_{w}-T_{\infty}\right) \\
& \text { and } S h_{x}=\frac{x q_{m}}{D_{B}\left(C_{w}-C_{\infty}\right)},
\end{aligned}
$$

Where $q_{w}$ and $q_{m}$ are heat and mass flux of the wall at the surface sheet correspondingly are specified by

$$
q_{w}=\left(-\left(k+\frac{16 \sigma^{*} T_{\infty}^{3}}{3 k^{*}}\right)\left(\frac{\partial T}{\partial y}\right)\right)_{y=0}, q_{m}=-D_{m}\left(\frac{\partial C}{\partial y}\right)_{y=0} .
$$

Finally replacing $\tau_{w}, q_{w}$ and $q_{m}$ in the Eqs. (15)-(17), we get

$$
\begin{aligned}
& \operatorname{Re}_{x}^{1 / 2} C f_{x}=\left(1+\frac{1}{\beta}\right) f^{\prime \prime}(0) /\left(\frac{2}{n+1}\right)^{\frac{1}{2}} \\
& \operatorname{Re}_{x}^{1 / 2} N u_{x}=-\left(1+\frac{4 R}{3}\right) \theta^{\prime}(0) /\left(\frac{2}{n+1}\right)^{\frac{1}{2}}, \\
& \operatorname{Re}_{x}^{1 / 2} S h_{x}=-\left(\frac{2}{n+1}\right)^{\frac{-1}{2}} \phi^{\prime}(0),
\end{aligned}
$$

Where the local Reynolds number is $\operatorname{Re}_{x}=\left(\frac{x U_{w}}{v}\right)^{2}$.

\section{Problem Solution Procedure}

The ordinary differential Eqs. (11) - (13) are non-linear and it is not possible to get closed-form solutions, so that, which have been numerically solved by implementing the Keller-Box technique and considering the boundary conditions mentioned in Eq. (14), The approach was developed by Keller, and considered to be very 
useful for working with non-linear problems and simply apply to any order. The major points in this method to achieve numerical solutions are:

i. $\quad$ Transform the given first-order differential equations into a linear set of equations.

ii. Write the converted ODE's in the form of finite differences.

iii. With Newton's method, the algebraic equations are linearized and put the equations into a matrix form.

iv. Lastly, to get the solution of the set of linear equations employs the block tri-diagonal elimination process.

\subsection{Keller-Box scheme}

The transformed differential equations (11)-(13) along with the boundary conditions (14) are numerically solved by Implicit finite-difference method known as the Keller-Box scheme which involves the following steps. Equations (11)-(13) are initially changed into a set of first ODE's, and by implementing central differences can be articulated to the difference equations.

\section{i) Finite difference scheme}

For this intention and introduce new variables $\chi(\eta), \xi(\eta), \theta(\eta), \Theta(\eta), \phi(\eta)$ and $w(\eta)$ with

$f^{\prime}=\chi, \chi^{\prime}=\xi, \theta^{\prime}=\Theta$ and $\phi^{\prime}=w$ so that the equations (11)-(13) are changed as

$f^{\prime}=\chi$

$\chi^{\prime}=\xi$

$\theta^{\prime}=\Theta$

$\phi^{\prime}=w$

$\xi^{\prime}+\left(\frac{\beta}{\beta+1}\right)\left(f \xi-\left(\frac{2}{n+1}\right)\left(n \cdot \chi^{2}-G r \cdot \theta+G c \cdot \phi \cdot \cos \alpha\right)-H a \cdot \chi\right)=0$

$\left(1+\frac{4 R}{3}\right) \Theta^{\prime}+\operatorname{Pr}\left(f v+N b \cdot w \Theta+N t \cdot \Theta^{2}+\left(\frac{\beta+1}{\beta}\right) E c \cdot \xi^{2}+Q \cdot \theta+J \cdot \chi^{2}\right)=0$

$w^{\prime}+S c \cdot(f w)+\frac{N t}{N b} \Theta^{\prime}-S c \cdot \gamma \cdot \phi=0$

The boundary conditions in Eq. (14) in terms of the new dependent variables, are given by

$$
\begin{aligned}
& \chi(0)=1, f(0)=S, \theta(0)=1, \phi(0)=1 \text { and } \\
& \chi(\eta) \rightarrow 0, \theta(\eta) \rightarrow 0, \phi(\eta) \rightarrow 0 \text { as } \eta \rightarrow \infty
\end{aligned}
$$

Now, consider the section $\eta_{j-1} \eta_{j}$, with $\eta_{j-1 / 2}$ as the centre point and which is characterized as below:

$x^{0}=0, x^{n}=x^{n-1}+k_{n}, n=1,2 \ldots . J$

$\eta_{j}=\eta_{j-1}+h_{j}, \eta_{j}=\eta_{\infty}, \eta_{0}=0, j=1,2 \ldots . . J$

Here $\left(h_{j}, k_{n}\right)$ is in the space $(\Delta \eta, \Delta x)$ and $n, j$ are equitable numbers in the sequence which indicates the position of the coordinates.

The differentials can be substituted with finite differences along the direction of $x$ the -axis, for instance at any given point the finite differences are as:

$$
\begin{aligned}
& ()_{j}^{n-\frac{1}{2}}=\frac{1}{2}\left[()_{j}^{n}+()_{j}^{n-1}\right]()_{j-\frac{1}{2}}^{n}=\frac{1}{2}\left[()_{j}^{n}+()_{j-1}^{n}\right] \\
& \left(\frac{\partial \chi}{\partial x}\right)_{j-1 / 2}^{n-\frac{1}{2}}=\frac{\left(\chi^{n}\right)_{j-\frac{1}{2}}-\left(\chi^{n-1}\right)_{j-\frac{1}{2}}}{k_{n}},\left(\frac{\partial \chi}{\partial \eta}\right)_{j-1 / 2}^{n-\frac{1}{2}}=\frac{\left(\chi^{n-1 / 2}\right)_{j}-\left(\chi^{n-1 / 2}\right)_{j-1}}{h_{j}}
\end{aligned}
$$


Now, express the finite difference structure of the equation for the center point $\left(x^{n}, \eta_{j-1 / 2}\right)$ by implementing the central difference derivatives. Equations (21)-(27) can be expressed in terms of the central point $\eta_{j-1 / 2}$ of the section $\eta_{j-1} . \eta_{j}$. This scheme provides

$$
\begin{aligned}
& f_{j}=\frac{h_{j}}{2}\left(\chi_{j}+\chi_{j-1}\right)+f_{j-1} \\
& \chi_{j}=\frac{h_{j}}{2}\left(\xi_{j}+\xi_{j-1}\right)+\chi_{j-1} \\
& \theta_{j}=\frac{h_{j}}{2}\left(\Theta_{j}+\Theta_{j-1}\right)+\theta_{j-1} \\
& \phi_{j}=\frac{h_{j}}{2}\left(w_{j}+w_{j-1}\right)+\phi_{j-1} \\
& \left(\frac{\beta+1}{\beta}\right)\left(\xi_{j}-\xi_{j-1}\right)+\left(f_{j}+f_{j-1}\right) \bullet\left(\xi_{j}+\xi_{j-1}\right) \bullet \frac{h_{j}}{4}- \\
& \left(\frac{2 h_{j}}{n+1}\right) \bullet\left(\frac{n}{4} \bullet\left(\chi_{j-1}+\chi_{j}\right)^{2}-\frac{G r}{2} \bullet\left(\theta_{j}+\theta_{j-1}\right)+\frac{G c}{2} \bullet\left(\phi_{j-1}+\phi_{j}\right) \cdot \cos \alpha\right)-h_{j} \frac{M}{2}\left(\chi_{j}+\chi_{j-1}\right)=0 \\
& \left(1+\frac{4 R}{3}\right)\left(\Theta_{j}-\Theta_{j-1}\right)+\frac{h_{j}}{4} \operatorname{Pr}\left(\left(f_{j}+f_{j-1}\right) \bullet\left(\Theta_{j}+\Theta_{j-1}\right)+N b\left(\Theta_{j}+\Theta_{j-1}\right) \bullet\left(w_{j}+w_{j-1}\right)\right) \\
& +\frac{h_{j}}{4} \operatorname{Pr}\left(N t\left(\Theta_{j}+\Theta_{j-1}\right)^{2}+\left(\frac{\beta+1}{\beta}\right) E c\left(\xi_{j}+\xi_{j-1}\right)^{2}+2 * Q\left(\theta_{j}+\theta_{j-1}\right)+J \cdot\left(\chi_{j}+\chi_{j-1}\right)^{2}\right)=0 \\
& w_{j}-w_{j-1}+\frac{h_{j}}{4} S c\left(f_{j}+f_{j-1}\right)\left(w_{j}+w_{j-1}\right)-\frac{N t}{N b}\left(\Theta_{j-1}-\Theta_{j}\right)-\frac{h_{j}}{2} S c \cdot \gamma\left(\phi_{j-1}+\phi_{j}\right)=0
\end{aligned}
$$

Equations (29)-(35) are inflicted for $\mathrm{j}=1,2,3 \ldots \mathrm{J}$, and also the altered thickness of the boundary layer $\eta_{J}$ to be adequately immense so that it is outside the boundary layer. Hence the conditions in Equation (28) are $f_{0}=S, \theta_{0}=1, \chi_{0}=1, \phi_{0}=1, \phi_{J}=0, \chi_{J}=0, \theta_{J}=0$

ii) Newton's method:

The set of Equations (29) - (35) are nonlinear to linearize these equations we adopt Newton's method, the following iterates are added.

$$
\left.\begin{array}{l}
\chi_{j}{ }^{(i+1)}=\left(\chi_{j}\right)^{i}+\left(\delta \chi_{j}\right)^{i}, \xi_{j}{ }^{(i+1)}=\left(\xi_{j}\right)^{i}+\left(\delta \xi_{j}\right)^{i}, w_{j}{ }^{(i+1)}=\left(w_{j}\right)^{i}+\left(\delta w_{j}\right)^{i}, \\
\Theta_{j}{ }^{(i+1)}=\left(\Theta_{j}\right)^{i}+\left(\delta \Theta_{j}\right)^{i}, \theta_{j}{ }^{(i+1)}=\left(\theta_{j}\right)^{i}+\left(\delta \theta_{j}\right)^{i}, \phi_{j}{ }^{(i+1)}=\left(\phi_{j}\right)^{i}+\left(\delta \phi_{j}\right)^{i} \text { and } f_{j}{ }^{(i+1)}=\left(f_{j}\right)^{i}+\left(\delta f_{j}\right)^{i}
\end{array}\right\}
$$

where $i=0,1,2, \ldots \ldots$

Substitute Eq. (37) in Equations (29)-(35) and then neglect the second and greater order quantities in $\delta f_{j}{ }^{i}, \delta \chi_{j}{ }^{i}, \delta \xi_{j}{ }^{i}, \delta \theta_{j}{ }^{i}, \delta \Theta_{j}{ }^{i}, \delta \phi_{j}{ }^{i}$ and $\delta w_{j}{ }^{i}$, this technique yields subsequent linear equations.

$\left(r_{1}\right)_{j-\frac{1}{2}}=\delta f_{j}-\delta f_{j-1}-\frac{h_{j}}{2}\left(\delta \chi_{j}+\delta \chi_{j-1}\right)$ 


$$
\begin{aligned}
& \left(r_{2}\right)_{j-\frac{1}{2}}=\delta \chi_{j}-\delta \chi_{j-1}-\frac{h_{j}}{2}\left(\delta \xi_{j}+\delta \xi_{j-1}\right) \\
& \left(r_{3}\right)_{j-\frac{1}{2}}=\delta \theta_{j}-\delta \theta_{j-1}-\frac{h_{j}}{2}\left(\delta \Theta_{j}+\delta \Theta_{j-1}\right) \\
& \left(r_{4}\right)_{j-\frac{1}{2}}=\delta \phi_{j}-\delta \phi_{j-1}-\frac{h_{j}}{2}\left(\delta w_{j}+\delta w_{j-1}\right) \\
& \left(r_{5}\right)_{j-\frac{1}{2}}=\left(e_{1}\right)_{j} \delta \xi_{j}+\left(e_{2}\right)_{j} \delta \xi_{j-1}+\left(e_{3}\right)_{j} \delta f_{j}+\left(e_{4}\right)_{j} \delta f_{j-1}+\left(e_{5}\right)_{j} \delta \chi_{j}+\left(e_{6}\right)_{j} \delta \chi_{j-1}+ \\
& \left(e_{7}\right)_{j} \delta \theta_{j}+\left(e_{8}\right)_{j} \delta \theta_{j-1}+\left(e_{9}\right)_{j} \delta \phi_{j}+\left(e_{10}\right)_{j} \delta \phi_{j-1} \\
& \left(r_{6}\right)_{j-\frac{1}{2}}=\left(d_{1}\right)_{j} \delta \Theta_{j}+\left(d_{2}\right)_{j} \delta \Theta_{j-1}+\left(d_{3}\right)_{j} \delta f_{j}+\left(d_{4}\right)_{j} \delta f_{j-1}+\left(d_{5}\right)_{j} \delta w_{j}+\left(d_{6}\right)_{j} \delta w_{j-1}+ \\
& \left(d_{7}\right)_{j} \delta \xi_{j}+\left(d_{8}\right)_{j} \delta \xi_{j-1}+\left(d_{9}\right)_{j} \delta \theta_{j}+\left(d_{10}\right)_{j} \delta \theta_{j-1}+\left(d_{11}\right)_{j} \delta \chi_{j}+\left(d_{12}\right)_{j} \delta \chi_{j-1} \\
& \left(r_{7}\right)_{j-\frac{1}{2}}=\left(g_{1}\right)_{j} \delta w_{j}+\left(g_{2}\right)_{j} \delta w_{j-1}+\left(g_{3}\right)_{j} \delta f_{j}+\left(g_{4}\right)_{j} \delta f_{j-1}+\left(g_{5}\right)_{j} \delta \Theta_{j}+\left(g_{6}\right)_{j} \delta \Theta_{j-1}+ \\
& \left(g_{7}\right)_{j} \delta \phi_{j}+\left(g_{8}\right)_{j} \delta \phi_{j-1}
\end{aligned}
$$

where

$$
\left.\begin{array}{l}
\left(e_{1}\right)_{j}=1+\frac{h_{j}}{2}\left(\frac{\beta}{\beta+1}\right) f_{j-\frac{1}{2}},\left(e_{2}\right)_{j}=\left(e_{1}\right)_{j}-2 \cdot 0,\left(e_{3}\right)_{j}=\frac{h_{j}}{2}\left(\frac{\beta}{\beta+1}\right) \xi_{j-\frac{1}{2}}=\left(e_{4}\right)_{j}, \\
\left(e_{5}\right)_{j}=-h_{j} \cdot\left(\frac{\beta}{\beta+1}\right) \cdot\left(\frac{2 n}{n+1}\right) \chi_{j-\frac{1}{2}}-\frac{h_{j}}{2} \cdot\left(\frac{\beta}{\beta+1}\right) \cdot H a=\left(e_{6}\right)_{j}, \\
\left(e_{7}\right)_{j}=h_{j} \cdot\left(\frac{\beta}{\beta+1}\right) \cdot\left(\frac{G r}{n+1}\right)=\left(e_{8}\right)_{j},\left(e_{9}\right)_{j}=-h_{j} \cdot\left(\frac{\beta}{\beta+1}\right) \cdot\left(\frac{G c}{n+1}\right) \cdot \cos \alpha=\left(e_{10}\right)_{j} \\
\left(d_{1}\right)_{j}=\left(1+\frac{4 R}{3}\right)+h_{j} \frac{\operatorname{Pr}}{2}\left(f_{j-\frac{1}{2}}+N b w_{j-\frac{1}{2}}+2 N t \cdot \Theta_{j-\frac{1}{2}}\right),\left(d_{2}\right)_{j}=\left(d_{1}\right)_{j}-2 \cdot 0, \\
\left(d_{3}\right)_{j}=h_{j} \frac{\operatorname{Pr}}{2} \Theta_{j-\frac{1}{2}}=\left(d_{4}\right)_{j},\left(d_{5}\right)_{j}=h_{j} \frac{\operatorname{Pr}}{2} N b \cdot \Theta \Theta_{j-\frac{1}{2}}=\left(d_{6}\right)_{j}, \\
\left(d_{7}\right)_{j}=h_{j} \frac{\operatorname{Pr}}{2} \cdot E c \cdot\left(\frac{\beta+1}{\beta}\right)_{j-\frac{1}{2}}=\left(d_{8}\right)_{j},\left(d_{9}\right)_{j}=h_{j} \frac{\operatorname{Pr}}{2} Q=\left(d_{10}\right)_{j},\left(d_{11}\right)_{j}=h_{j} \operatorname{Pr} \cdot J \cdot \chi_{j-\frac{1}{2}}=\left(d_{12}\right)_{j} \\
\left(g_{1}\right)_{j}=1+h_{j} \frac{S c}{2} f_{j-\frac{1}{2}},\left(g_{2}\right)_{j}=\left(g_{1}\right)_{j}-2 \cdot 0,\left(g_{3}\right)_{j}=h_{j} \frac{S c}{2} w_{j-\frac{1}{2}}=\left(g_{4}\right)_{j}, \\
\left(g_{5}\right)_{j}=\frac{N t}{N b},\left(g_{6}\right)_{j}=-\left(g_{5}\right)_{j},\left(g_{7}\right)_{j}=-S c \frac{h_{j}}{2} \gamma=\left(g_{8}\right)_{j}
\end{array}\right\}
$$


and

$\left(r_{1}\right)_{j-\frac{1}{2}}=f_{j-1}-f_{j}+h_{j} \chi_{j-\frac{1}{2}}$

$\left(r_{2}\right)_{j-\frac{1}{2}}=\chi_{j-1}-\chi_{j}+h_{j} \xi_{j-\frac{1}{2}}$

$\left(r_{3}\right)_{j-\frac{1}{2}}=\theta_{j-1}-\theta_{j}+h_{j} \Theta_{j-\frac{1}{2}}$

$\left(r_{4}\right)_{j-\frac{1}{2}}=\phi_{j-1}-\phi_{j}+h_{j} w_{j-\frac{1}{2}}$

$\left(r_{5}\right)_{j-\frac{1}{2}}=\xi_{j-1}-\xi_{j}-h_{j} \cdot\left(\frac{\beta}{\beta+1}\right)(f \xi)_{j-\frac{1}{2}}+$

$h_{j} \cdot\left(\frac{\beta}{\beta+1}\right) \cdot\left(\frac{2}{n+1}\right)\left[n \cdot\left(\chi^{2}\right)_{j-\frac{1}{2}}-G r \cdot \theta_{j-\frac{1}{2}}+G c \cdot \cos \alpha \cdot \phi_{j-\frac{1}{2}}\right]+h_{j} \cdot\left(\frac{\beta}{\beta+1}\right) \cdot H a \cdot \chi_{j-\frac{1}{2}}$

$\left(r_{6}\right)_{j-\frac{1}{2}}=\left(1+\frac{4 R}{3}\right)\left(\Theta_{j-1}-\Theta_{j}\right)-h_{j} \operatorname{Pr}\left[\begin{array}{l}(f \Theta)_{j-\frac{1}{2}}+N b(\Theta w)_{j-\frac{1}{2}}+N t\left(\Theta^{2}\right)_{j-\frac{1}{2}}+\left(\frac{\beta+1}{\beta}\right)\left(\xi^{2}\right)_{j-\frac{1}{2}} \\ +Q . \theta_{j-\frac{1}{2}}+J \cdot\left(\chi^{2}\right)_{j-\frac{1}{2}}\end{array}\right]$

$\left(r_{7}\right)_{j-\frac{1}{2}}=\left(w_{j-1}-w_{j}\right)-h_{j} S c(f w)_{j-\frac{1}{2}}-\frac{N t}{N b}\left(\Theta_{j}-\Theta_{j-1}\right)+S c \cdot h_{j} \cdot \gamma \cdot \phi_{j-\frac{1}{2}}$

Boundary conditions (36) that can be met exactly without iterations. Therefore, in order for these values to remain correct throughout all iterations, we take

$\delta f_{0}=0, \delta \chi_{0}=0, \delta \theta_{0}=0, \delta \phi_{0}=0, \delta \chi_{j}=0, \delta \theta_{j}=0, \delta \phi_{j}=0$

iii) Block-elimination method

The difference equations (38) - (44) are linear and have a structure of block tri-diagonal matrix usually it contains constants or variables and by using the block-elimination procedure, it can be solved efficiently, but it can be noted here that an important aspect is made up of block matrices. Equations (38)-(44) are in a matrixvector form as

A. $\delta=\mathrm{r}$

$\left[\begin{array}{lll}A_{1} & C_{1} & \\ B_{2} & A_{2} & C_{2}\end{array}\right.$

The components in matrices are given below: 


$$
\begin{aligned}
& {\left[\begin{array}{ccccrrr}
0 & 0 & 0 & 1 & 0 & 0 & 0 \\
-\frac{h_{j}}{2} & 0 & 0 & 0 & -\frac{h_{j}}{2} & 0 & 0
\end{array}\right]} \\
& {\left[A_{1}\right]=\left[\begin{array}{ccccccc}
0 & -\frac{h_{j}}{2} & 0 & 0 & 0 & -\frac{h_{j}}{2} & 0 \\
0 & 0 & -\frac{h_{j}}{2} & 0 & 0 & 0 & -\frac{h_{j}}{2} \\
\left(e_{2}\right)_{j} & 0 & 0 & \left(e_{3}\right)_{j} & \left(e_{1}\right)_{j} & 0 & 0 \\
\left(d_{8}\right)_{j} & \left(d_{2}\right)_{j} & \left(d_{6}\right)_{j} & \left(d_{3}\right)_{j} & \left(d_{7}\right)_{j} & \left(d_{1}\right)_{j} & \left(d_{5}\right)_{j} \\
0 & \left(g_{6}\right)_{j} & \left(g_{2}\right)_{j} & \left(g_{3}\right)_{j} & 0 & \left(g_{5}\right)_{j} & \left(g_{1}\right)_{j}
\end{array}\right]} \\
& {\left[A_{J}\right]=\left[\begin{array}{ccccccc}
-\frac{h_{j}}{2} & 0 & 0 & 1 & 0 & 0 & 0 \\
-1 & 0 & 0 & 0 & -\frac{h_{j}}{2} & 0 & 0 \\
0 & -1 & 0 & 0 & 0 & -\frac{h_{j}}{2} & 0 \\
0 & 0 & -1 & 0 & 0 & 0 & -\frac{h_{j}}{2} \\
\left(e_{6}\right)_{j} & \left(e_{8}\right)_{j} & \left(e_{10}\right)_{j} & \left(e_{3}\right)_{j} & \left(e_{1}\right)_{j} & 0 & 0 \\
\left(d_{12}\right)_{j} & \left(d_{10}\right)_{j} & 0 & \left(d_{3}\right)_{j} & \left(d_{7}\right)_{j} & \left(d_{1}\right)_{j} & \left(d_{5}\right)_{j} \\
0 & 0 & \left(g_{8}\right)_{j} & \left(g_{3}\right)_{j} & 0 & \left(g_{5}\right)_{j} & \left(g_{1}\right)_{j}
\end{array}\right], 2 \leq j \leq J} \\
& {\left[B_{J}\right]=\left[\begin{array}{ccccccc}
0 & 0 & 0 & -1 & 0 & 0 & 0 \\
0 & 0 & 0 & 0 & -\frac{h_{j}}{2} & 0 & 0 \\
0 & 0 & 0 & 0 & 0 & -\frac{h_{j}}{2} & 0 \\
0 & 0 & 0 & 0 & 0 & 0 & -\frac{h_{j}}{2} \\
0 & 0 & 0 & \left(e_{4}\right)_{j} & \left(e_{2}\right)_{j} & 0 & 0 \\
0 & 0 & 0 & \left(d_{4}\right)_{j} & \left(d_{8}\right)_{j} & \left(d_{2}\right)_{j} & \left(d_{6}\right)_{j} \\
0 & 0 & 0 & \left(g_{4}\right)_{j} & 0 & \left(g_{6}\right)_{j} & \left(g_{2}\right)_{j}
\end{array}\right], 2 \leq j \leq J-1} \\
& {\left[C_{J}\right]=\left[\begin{array}{ccccccc}
-\frac{h_{j}}{2} & 0 & 0 & 0 & 0 & 0 & 0 \\
1 & 0 & 0 & 0 & 0 & 0 & 0 \\
0 & 1 & 0 & 0 & 0 & 0 & 0 \\
0 & 0 & 1 & 0 & 0 & 0 & 0 \\
\left(e_{5}\right)_{j} & \left(e_{7}\right)_{j} & \left(e_{9}\right)_{j} & 0 & 0 & 0 & 0 \\
\left(d_{11}\right)_{j} & \left(d_{9}\right)_{j} & 0 & 0 & 0 & 0 & 0 \\
0 & 0 & \left(g_{7}\right)_{j} & 0 & 0 & 0 & 0
\end{array}\right], 1 \leq j \leq J-1}
\end{aligned}
$$

and 
$\left[\delta_{1}\right]=\left[\begin{array}{c}\delta \xi_{0} \\ \delta v_{0} \\ \delta w_{0} \\ \delta f_{1} \\ \delta \xi_{1} \\ \delta \Theta_{1} \\ \delta w_{1}\end{array}\right], \quad\left[\delta_{j}\right]=\left[\begin{array}{c}\delta \chi_{j-1} \\ \delta \theta_{j-1} \\ \delta \phi_{j-1} \\ \delta f_{j} \\ \delta \xi_{j} \\ \delta \Theta_{j} \\ \delta w_{j}\end{array}\right] 2 \leq j \leq J$, and $\left[r_{j}\right]=\left[\begin{array}{c}\left(r_{1}\right)_{j-\frac{1}{2}} \\ \left(r_{2}\right)_{j-\frac{1}{2}} \\ \left(r_{3}\right)_{j-\frac{1}{2}} \\ \left(r_{4}\right)_{j-\frac{1}{2}} \\ \left(r_{5}\right)_{j-\frac{1}{2}} \\ \left(r_{6}\right)_{j-\frac{1}{2}} \\ \left(r_{7}\right)_{j-\frac{1}{2}}\end{array}\right] 1 \leq j \leq J$

to solve Equation (47), consider that $\mathrm{A}$ is not a singular matrix and now express $\mathrm{A}$ as

$\mathrm{A}=\mathrm{L} . \mathrm{U}$

where $L=\left[\begin{array}{cccc}{\left[\alpha_{1}\right]} & & & \\ {\left[\beta_{2}\right]} & {\left[\alpha_{2}\right]} & & \\ & \ddots & \\ & \ddots & {\left[\alpha_{J-1}\right]} \\ & & {\left[\beta_{J}\right]} & {\left[\alpha_{J}\right]}\end{array}\right]$ and $U=\left[\begin{array}{cccc}{[I]} & {\left[\Gamma_{1}\right]} & & \\ & {[I]} & & \\ & \ddots & \\ & \ddots & {[I]} & {\left[\Gamma_{J-1}\right]} \\ & & {[I]}\end{array}\right]$ where $\left[\alpha_{i}\right]$ and

$\left[\Gamma_{i}\right]$ both are $7^{\text {th }}$ order square matrices and $[I]$ is a $7^{\text {th }}$ rowed square matrix which components are obtained by below equations:

$\left[\alpha_{1}\right]=\left[A_{1}\right]$

$\left[A_{1}\right]\left[\Gamma_{1}\right]=\left[C_{1}\right]$

$\left.\left\lfloor\alpha_{j}\right\rfloor=\left\lfloor A_{j}\right\rfloor-\left\lfloor B_{j}\right\rfloor \Gamma_{j-1}\right\rfloor ; j=2,3 \ldots . . J$

$\left.\left\lfloor\alpha_{j}\right\rfloor \Gamma_{j}\right\rfloor=\left\lfloor C_{j}\right\rfloor ; j=2,3 \ldots \ldots J-1$

After substituting Equation (48) in Eq. (47) becomes,

$L U \delta=r$

Let $W$ defined as $W=U \bullet \delta$

Then Eq. (53) turns into

$L W=r$

Where $W=\left[\begin{array}{c}{\left[W_{1}\right]} \\ {\left[W_{2}\right]} \\ \vdots \\ {\left[W_{j-1}\right]} \\ {\left[W_{j}\right]}\end{array}\right] ; 1 \leq j \leq J$ and $\left[W_{j}\right\rfloor$ are column matrices of order $7 \times 1$. The element of $W$ can be determined from Eq. (54) by the below relations.

$\left.\left.\left[\alpha_{1}\right]\left[W_{1}\right]=\left[r_{1}\right],\left\lfloor\alpha_{j}\right\rfloor W_{j}\right\rfloor=\left\lfloor r_{j}\right\rfloor-\left\lfloor B_{j}\right\rfloor W_{j-1}\right\rfloor ; 2 \leq j \leq J$

In the computation of $\alpha_{j}, W_{j}$ and $\Gamma_{j}$ the step is generally indicated as forward sweep. When the element of

$W$ has been found, Eq. (54) provides a result $\delta$ in the reverse sweep, here the components are achieved under the relations given below:

$\left.\left[\delta_{J}\right]=\left[W_{J}\right],\left\lfloor\delta_{j}\right\rfloor=\left\lfloor W_{j}\right\rfloor-\left\lfloor\Gamma_{j}\right\rfloor \delta_{j+1}\right\rfloor, 1 \leq j \leq J-1$

These computations are replicated up till a certain criterion of convergence is fulfilled and then stop calculations when

$\left|\delta \xi_{0}^{(i)}\right| \leq \varepsilon_{1},\left(\varepsilon_{1}<<0\right)$

Thermal radiation and joule heating effects on a magnetohydrodynamic Casson Nanofluid flow in the presence of chemical ... 
The current approach is unconditionally reliable, second-order consistency, and simple to program, making its output extremely desirable. The adaption of the related initial guessing is one of the variables that alter the consistency of the scheme. The consistency of the treatment depends on the right decision. Based on the convergence requirements and fulfill the boundary constraints (14), the acceptable guesses for the initial assumptions are:

$$
f(\eta)=1+S-e^{-\eta}, \theta(\eta)=e^{-\eta}, \phi(\eta)=e^{-\eta}
$$

The present study consists of a uniform grid size $\Delta \eta=0.005$ that gives four decimal places of precision in most of the specified values as seen in the table with the error of tolerance $10^{-5}$ in any situation.

\section{Results and Discussion}

The partial differential equations (2), (3), (5) and (8) along with the boundary conditions (9) are transformed into a set of ordinary differential equations by using the similarity transformations (10). The converted Eqs. (11) to (13) subject to the constraints (14) are numerically solved by utilizing MATLAB software with the help of Keller Box technique. In the present analysis, the following parameter values are adopted for computations: $\mathrm{Gr}$ $=\mathrm{Gc}=1.0, \mathrm{~S}=\mathrm{n}=\mathrm{Ha}=0.5, \mathrm{Pr}=0.72, \mathrm{Ec}=0.3, \mathrm{Q}=0.2, \beta=0.5, \alpha=\frac{\pi}{6}, \gamma=1.5, \mathrm{Sc}=0.3, \mathrm{R}=\mathrm{J}=0.5, \mathrm{Nt}=\mathrm{Nb}$ $=0.3$. Obtained results of the impact of variations in the values of different parameters on velocity, temperature, and concentration profiles and their influence on skin friction coefficient, local Nusselt and Sherwood numbers are described graphically.

First, in Figs. 2 to 4, the impact on velocity, temperature, and concentration distributions is shown respectively by the Casson fluid parameter. In Fig. 2 , the velocity has decreased by increasing $\beta$. Enhancing $\beta$ mechanically allows fluid viscosity to rise so it is apparent that the increased viscosity outcomes in a smaller velocity. Furthermore, from Figs. 3 and 4, it is found that the same increase in viscosity contributed to the enhances in the temperature of the nanofluid and decrease in the concentration profiles.

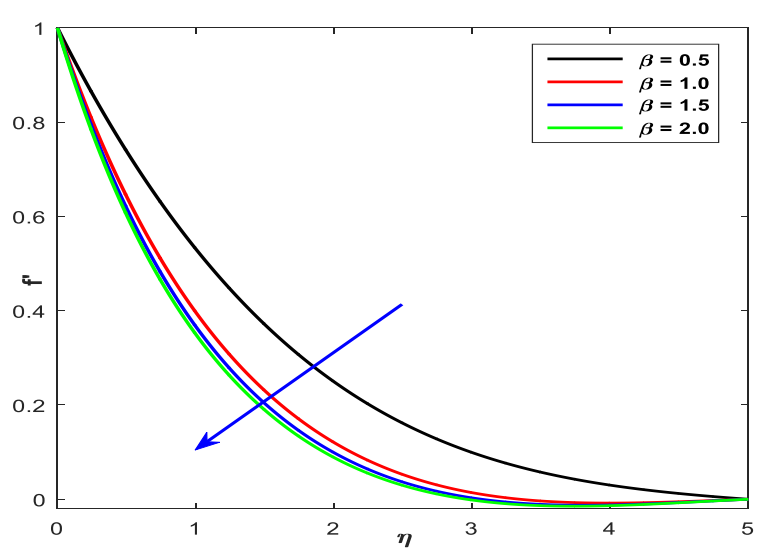

Fig. 2: Variation of $\beta$ on Velocity

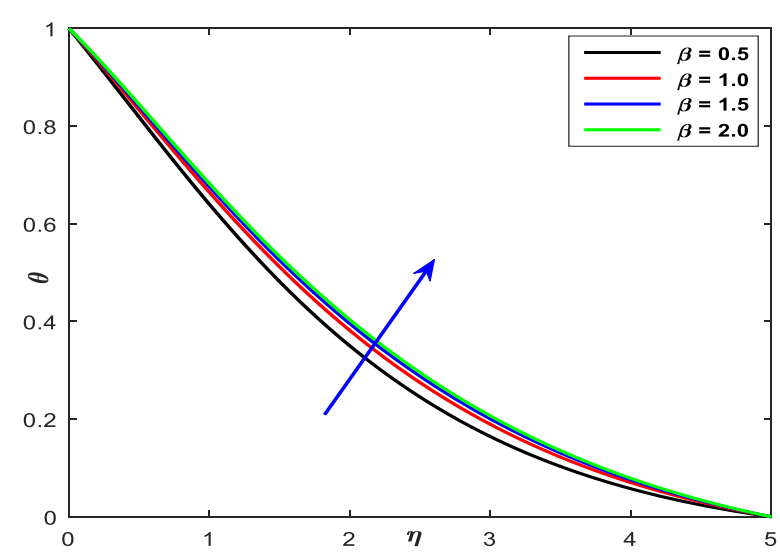

Fig. 3: Variation of $\beta$ on Temperature.

Fig. 5 indicates the impact of the angle of inclination on the velocity curves. It is known that the increased values of $\alpha$ raise the effect in the velocity curves. The influence on the fluid temperature of the inclination is given with the aid of Fig. 6. It is found that the temperature decreases with higher estimates of inclination.

Fig. 7 demonstrates the behavior of rising inclination values on the distribution of fluid concentration. It is found that the concentrations decline as the angle of inclination rises. Fig. 8 demonstrates the velocity graph influences of the Eckert number $(E c)$. Consequently, the surface velocity increases with increasing Eckert number. Fig. 9 elucidates the influence of the Ec on temperature. Due to heat applied by friction heating, Ec raises the wall temperature. The impact of the Joule heating parameter on the velocity and temperature distributions is presented in Figs. 10 and 11. It is initiate that the momentum and temperature curves are raises with the enhancing values of Joule heating $(\mathrm{J})$. 


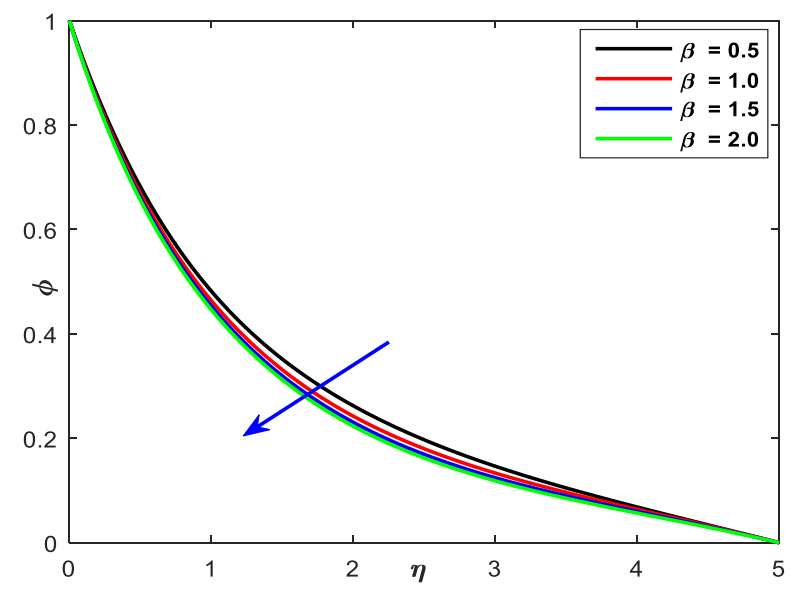

Fig. 4: Variation of $\beta$ on Concentration.

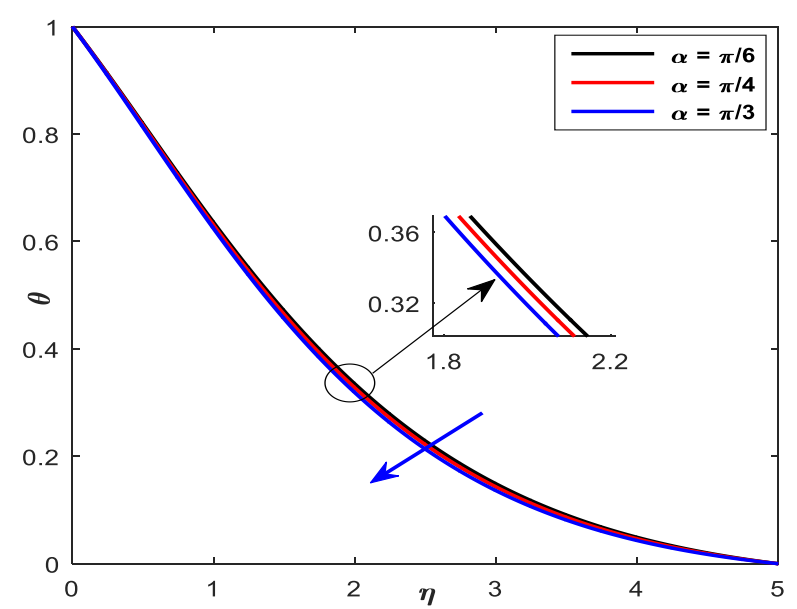

Fig. 6: Variation of $\alpha$ on Temperature.

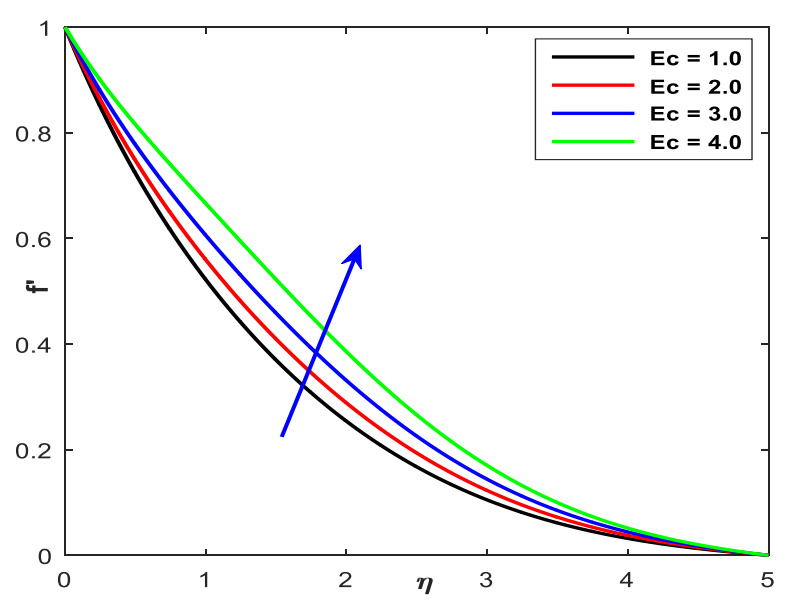

Fig. 8: Variation of Ec on Velocity.

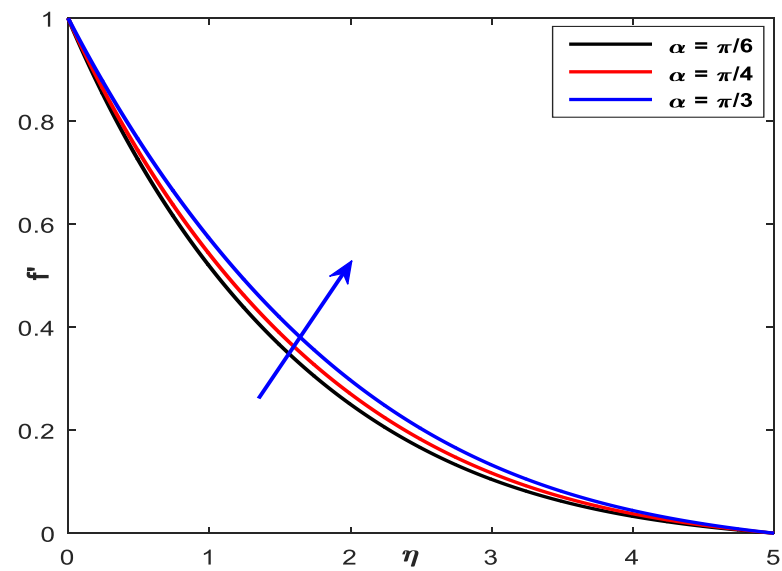

Fig. 5: Variation of $\alpha$ on Velocity.

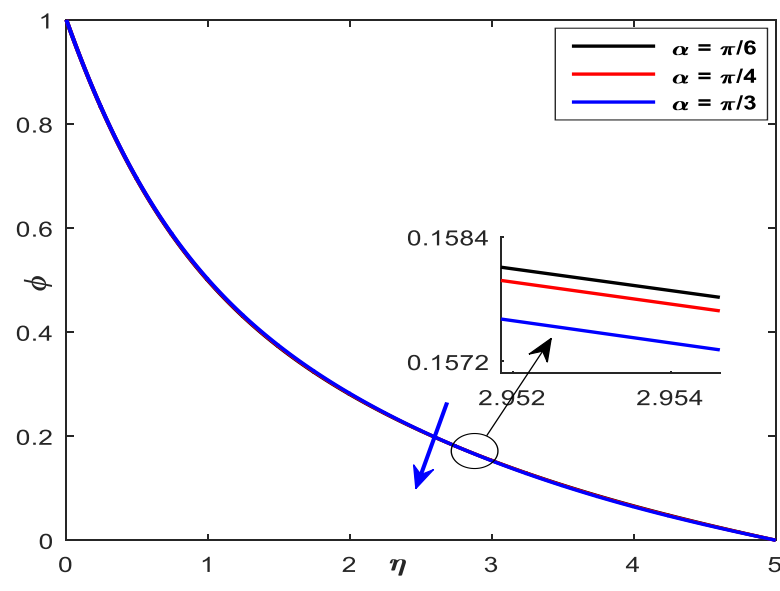

Fig. 7: Variation of $\alpha$ on Concentration.

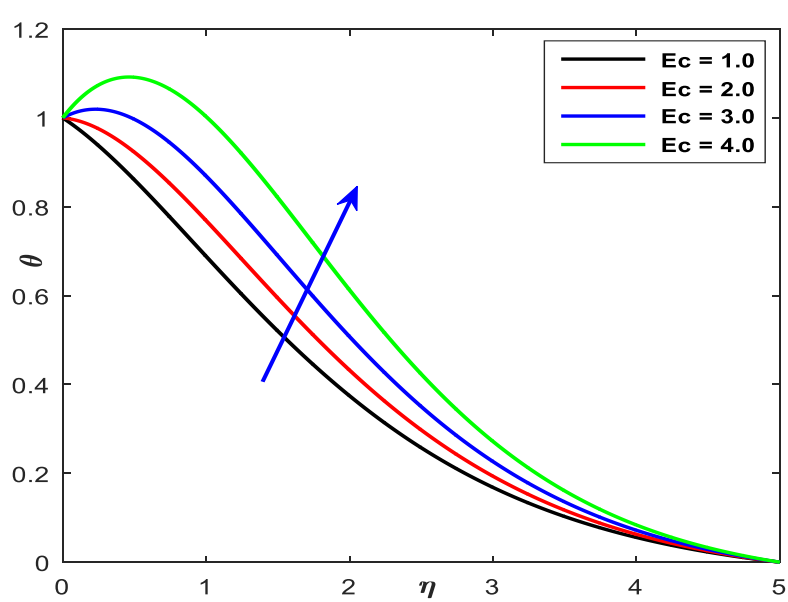

Fig. 9: Variation of Ec Temperature. 


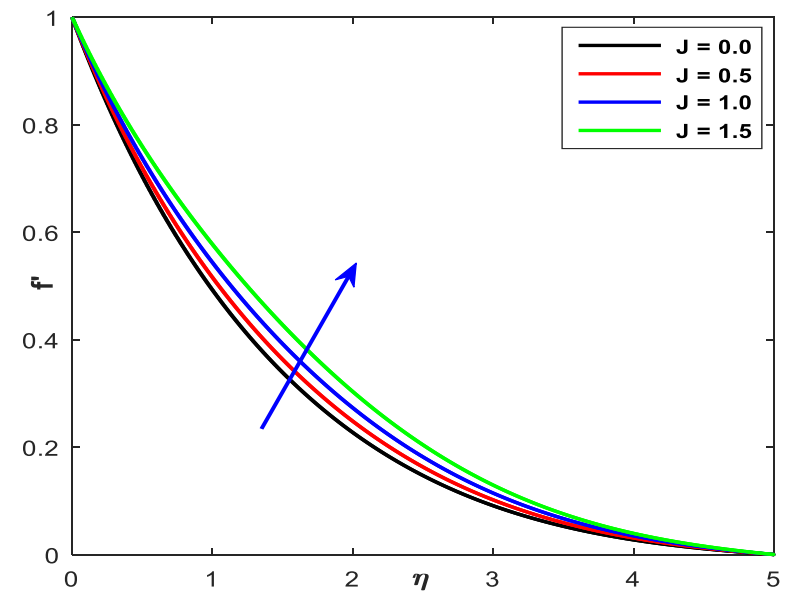

Fig. 10: Variation of $\mathrm{J}$ on Velocity

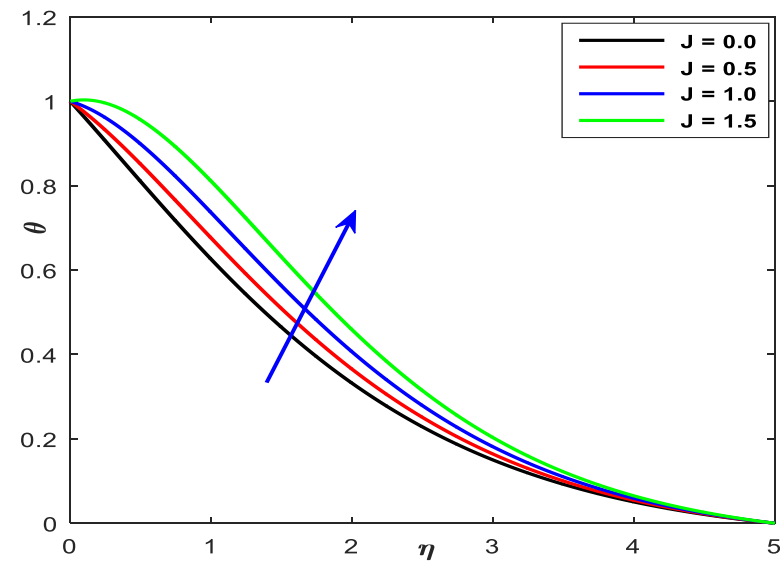

Fig. 11: Variation of $\mathrm{J}$ on Temperature.

Figs. 12-14 shows the influence of the mass transfer coefficient $S(S>=0)$ on dimensionless velocity, temperature and concentration. From Fig. 12, It shows the impact of the suction on fluid velocity, increasing suction parameter $S$ magnitude, which is observed to lower the velocity distribution. The temperature and concentration distribution profile of the augmented suction parameter $S$ is shown in Figs. 13 and 14. From these figures, it is evident that with the inclusion of the suction parameter, the thickness of the thermal boundary layer as well as the concentration declines.

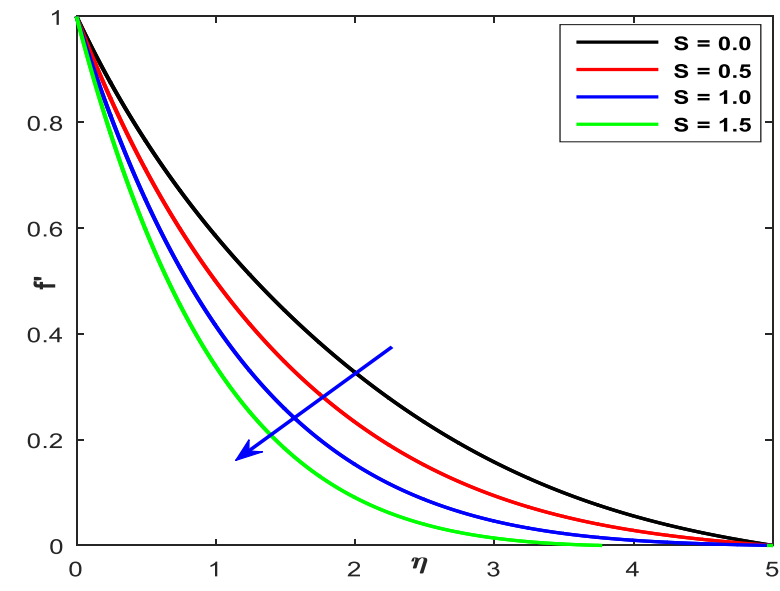

Fig.12: Variation of S on Velocity

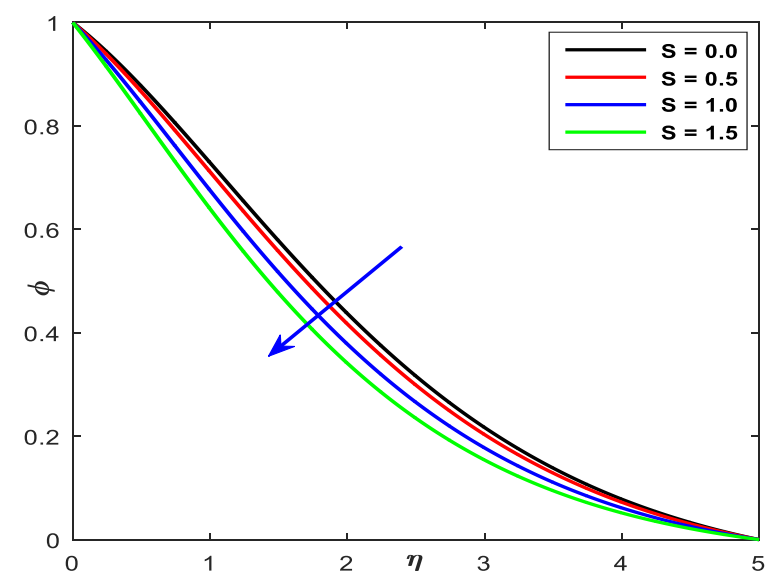

Fig. 14: Variation of S on Concentration

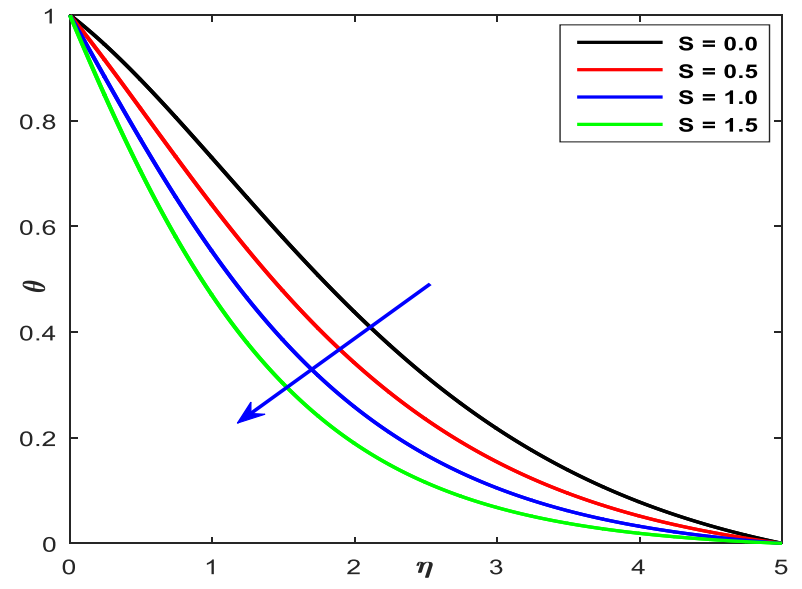

Fig. 13: Variation of S on Temperature.

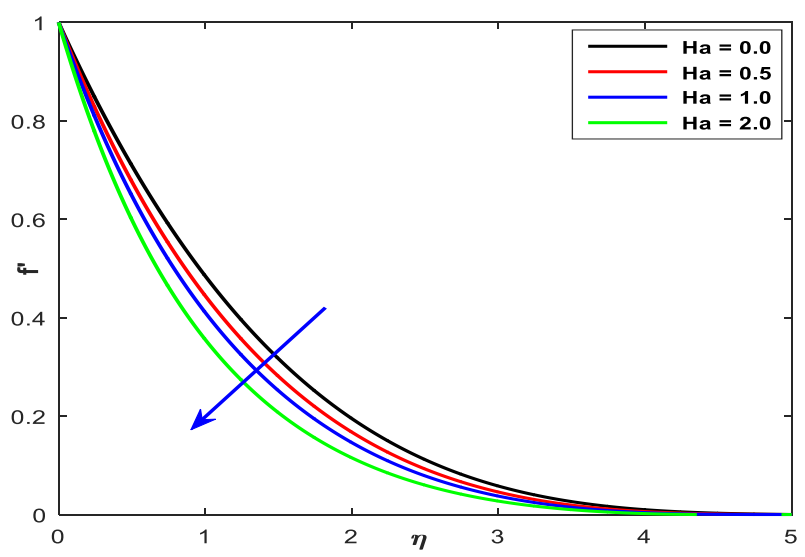

Fig.15: Variation of Ha on Velocity. 
Figs. 15 and 16 demonstrates that higher magnetic variable Ha values lead to a greater thermal field and thickness of the corresponding layer. Physically, as a result of the higher magnetic parameter that produces fluid motion resistance and therefore transforms some valued energy into heat, the Lorentz force improves. This results in an enhance in the temperature field.

The temperature curves are influenced by thermal radiation as shown in Fig. 17. The temperature has been shown to raise with the radiation parameter $\mathrm{R}$. This is compatible with the empirical observation that the thermal boundary width rises as $\mathrm{R}$ increases. Fig. 18 shows the effects on the temperature curves of the heat source factor. Since the parameter of the heat source in the system leads to heat generation, the temperature profile is expected to rise as the heat source factor increases. The temperature profile behavior is evident in the figure. with a difference in heat source factor. Fig. 19 displays the changes in the concentration when Brownian motion factor $\mathrm{Nb}$ is changed. The thickness of the concentric limit layer decreases with the rising values of $\mathrm{Nb}$. In comparison, with the $\mathrm{Nb}$ values rising. the amount of the concentration gradient on a sheet surface increases.

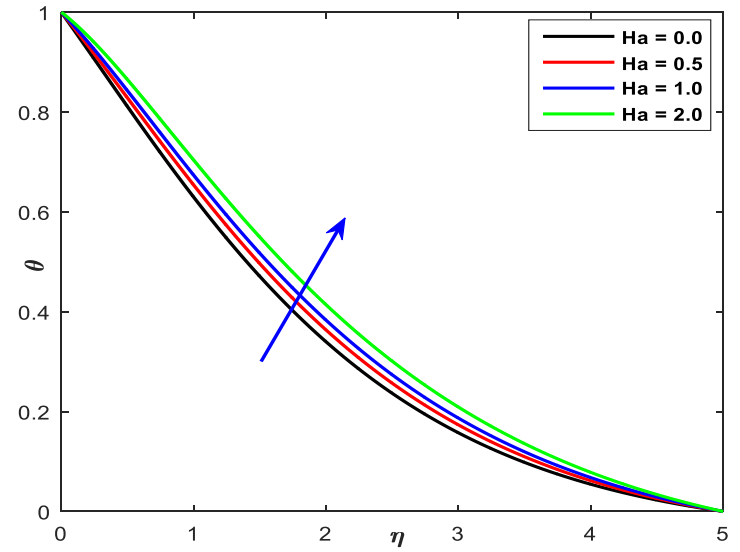

Fig. 16: Variation of Ha on Temperature.

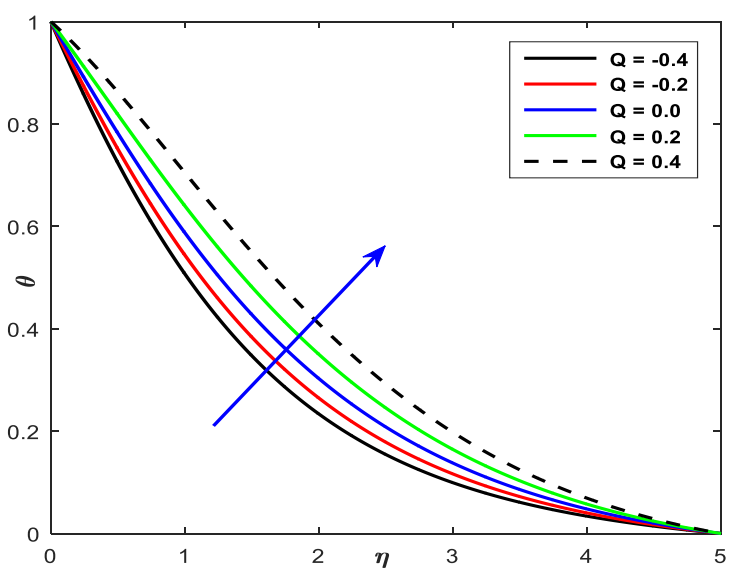

Fig. 18: Variation of Q on Temperature

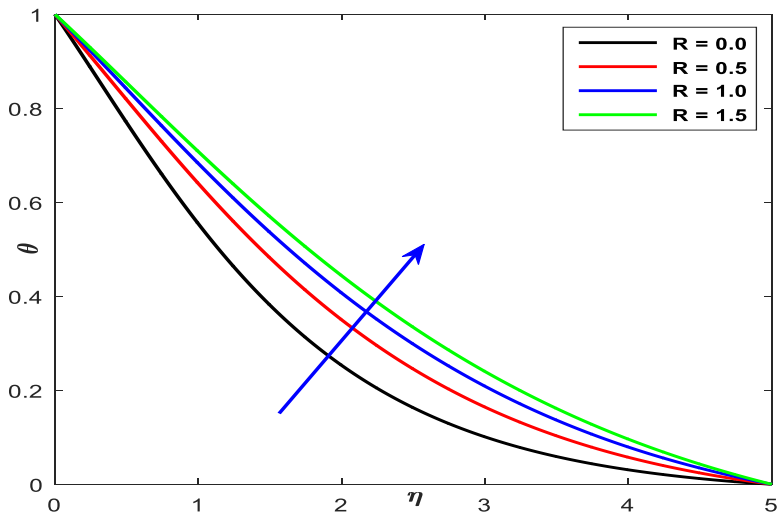

Fig.17: Variation of R on Temperature.

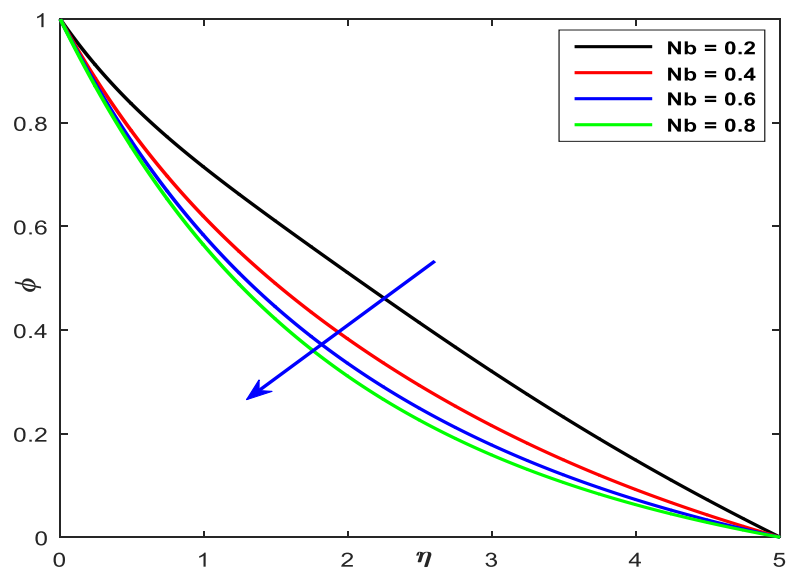

Fig.19: Variation of $\mathrm{Nb}$ on Concentration

The rise of the thermal field and corresponding thickness layer for bigger $N t$ are clearly seen in Fig. 20. Heated elements are drawn away from the hot area to the cool zone during thermophoresis. This is because the temperature of the fluid is increasing. Fig. 21 indicates the reduction in concentration as the chemical reaction factor $\gamma$ rises. This refers that the destructive reaction occurs in reductions in the area of concentration that fail to yield buoyancy effects as a function of gradients of concentration. Fig. 22 demonstrates the influence of ' $H a^{\prime}$ in the occurrence of ' $n$ ' on the skin friction factor. It is understood that with the frequency of ' $n$ ', the outward drag force is enhanced as ' $H a^{\prime}$ is enhancing. This was effectively induced by the Lorentz force and friction parameter of the magnetic field on the stream path and the high flux pressure.

Fig. 23 also demonstrates the close relationship between the friction coefficient with $S$ and $\beta$. Skin friction enhances with increasing the values of $S$ in the existence of $n$. The basic relation between the local Nusselt and 
$R$ in Fig. 24 is clear in the existence of $n$. The heat transmission rate mechanically is improved by $R$ and $n$, respectively, with the change of high heat molecular strength and high flow density. Likewise, the local number of Nusselt has a close correlation to the Grand $n$ in Fig. 25, with an increase of Gr the local Nusselt number increases. Finally, the significant connection among local Sherwood number and $\gamma$ is seen in the occurrence of $n$ as depicted in Fig. 26. The effect is induced by an improved mass transfer rate when the chemical parameter is changed.

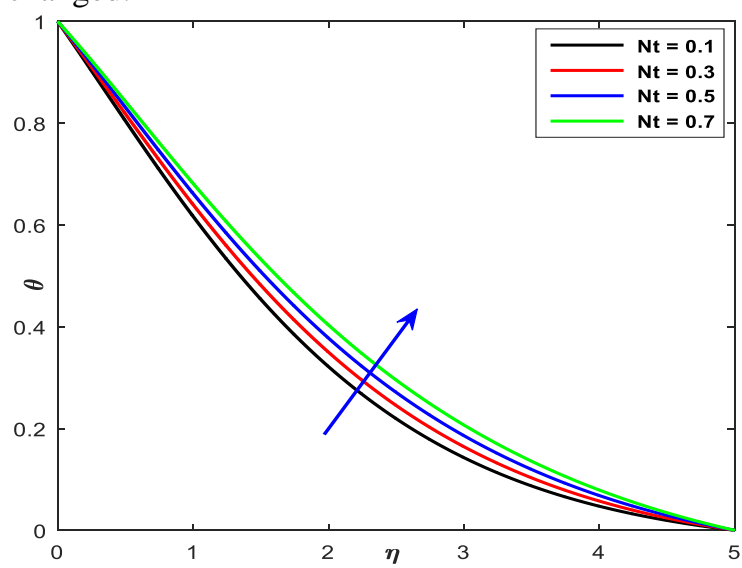

Fig. 20: Variation of $\mathrm{Nt}$ on Temperature

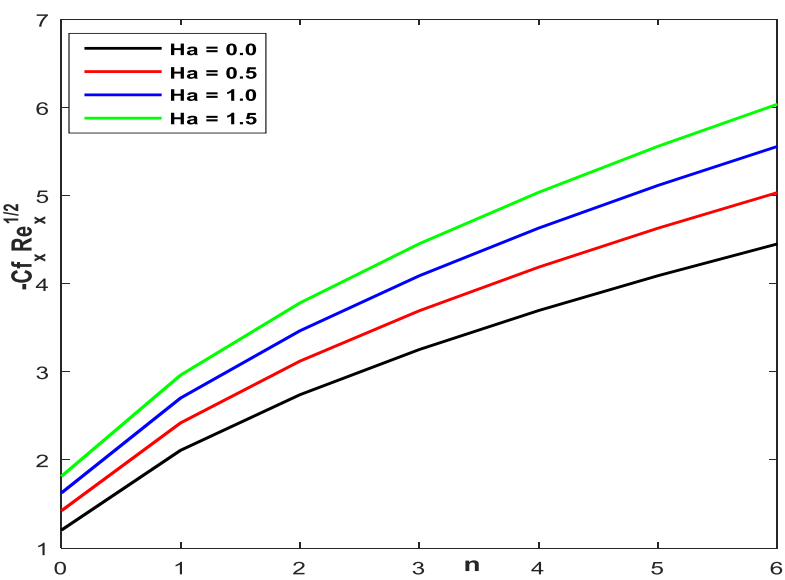

Fig. 22: Variation of Ha and $\mathrm{n}$ on skin friction coefficient

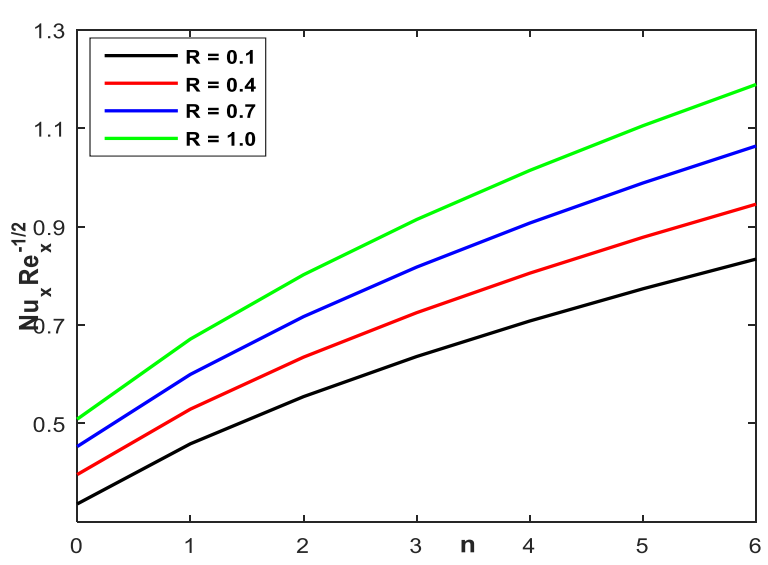

Fig. 24: Variation of $\mathrm{R}$ and $\mathrm{n}$ on local Nusselt number.

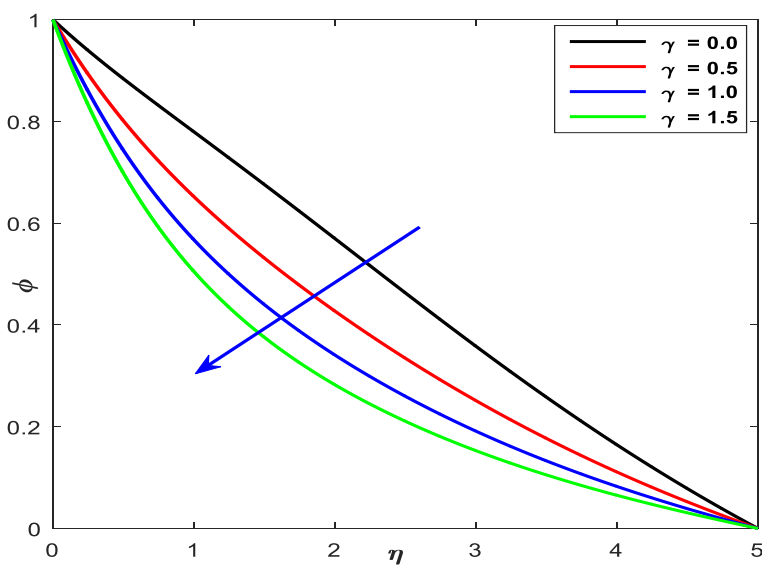

Fig. 21: Variation of $\gamma$ on Concentration

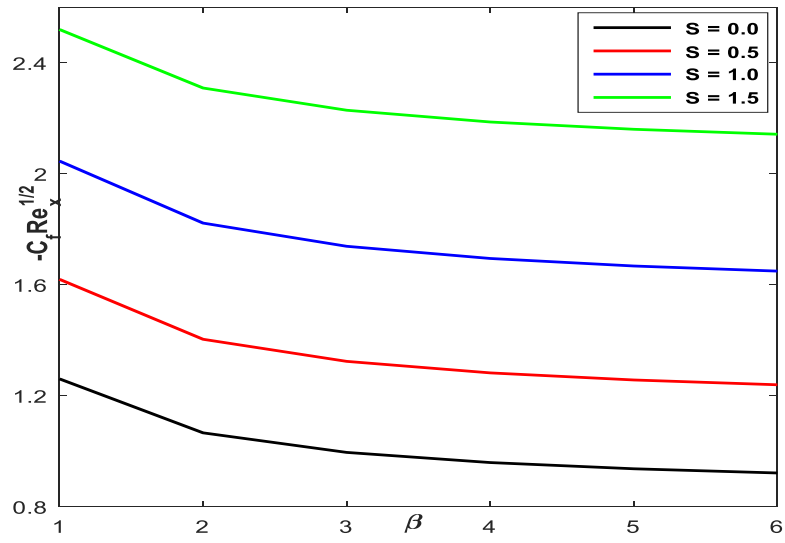

Fig. 23: Variation of $\mathrm{S}$ and $\beta$ on the skin friction coefficient

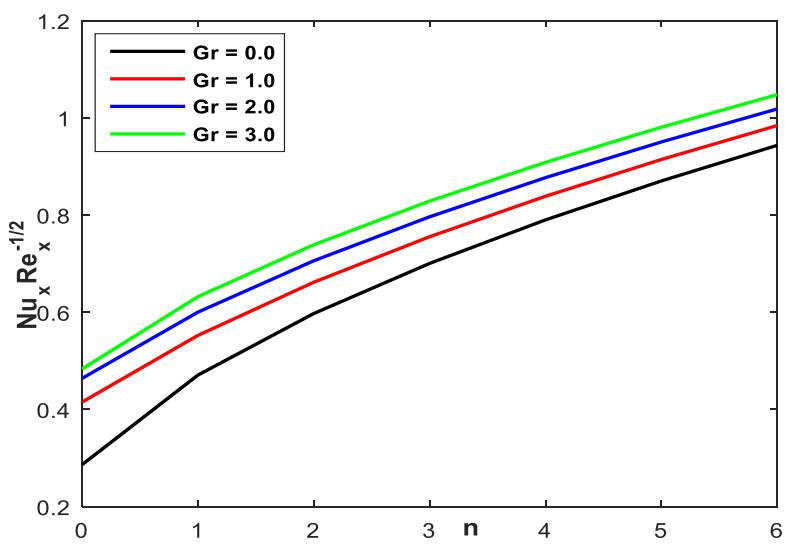

Fig. 25: Variation of Gr and $\mathrm{n}$ on local Nusselt number. 


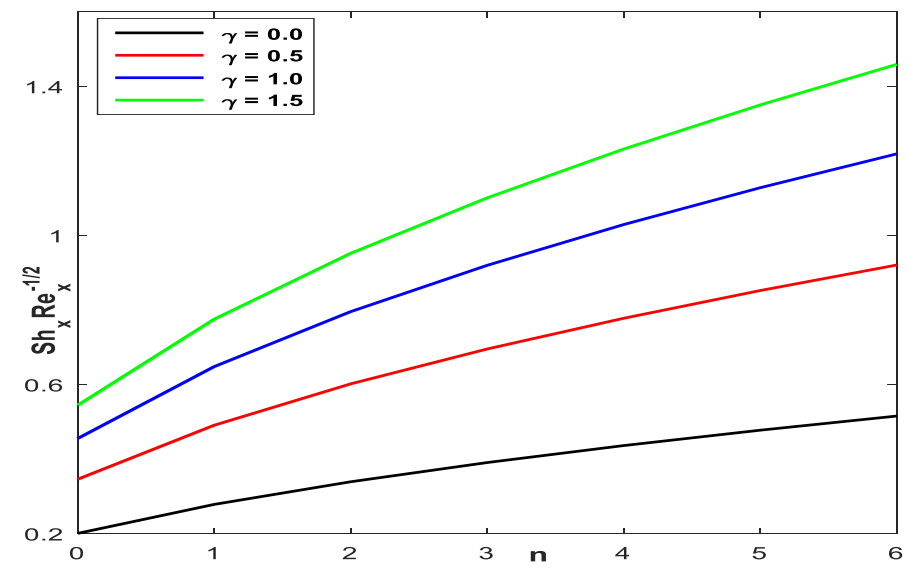

Fig. 26: Variation of $\gamma$ and $n$ on local Sherwood number

Table 1 demonstrates the admirable relation between the current findings and the previously reported results for the local Nusselt and Sherwood figures of Nadeem et al. (2014), Ibrahim et al. (2017) and Liao (2011). The consequence of many related parameters such as the quantity of skin friction, Table 2 presents local Nusselt and local Sherwood number. Table 2 reveals that the coefficient of skin friction, rate of heat transfer, and mass transfer drops with an enhanced Casson fluid parameter. This happens when viscous forces are dominated by the flux of which Casson fluid values. Both the Skin friction factor and Nusselt number depreciate with $J, Q$ and $\alpha$ although the rate of mass transfer enhances. The enhanced values of $J, Q$ and $\alpha$ produces the low pressure and intermolecular forces in the flow correspondingly. Nusselt and Sherwood number depreciate with Ha, whereas the Skin friction coefficient accelerates. Skin friction coefficient boosts with $G c$ and reduce velocity with $G r$, while Sherwood number and Nusselt number accelerates with $G r$ and falls with $G c$.

Table 1: Assessment of $-\theta^{\prime}(0)$ and $-\phi^{\prime}(0)$ when $G c=G r=H a=E c=S=R=Q=J=\gamma=\alpha=0, \operatorname{Pr}=S c$ $=2.0, N b=0.5$ and $\beta \rightarrow \infty$.

\begin{tabular}{|c|c|c|c|c|c|c|c|c|c|}
\hline \multirow{2}{*}{$\mathrm{N}$} & \multirow{2}{*}{$\mathrm{Nt}$} & \multicolumn{4}{|c|}{$\begin{array}{l}\text { Nusselt number } \\
\qquad-\theta^{\prime}(0)\end{array}$} & \multicolumn{4}{|c|}{$\begin{array}{c}\text { Sherwood number } \\
-\phi^{\prime}(0)\end{array}$} \\
\hline & & $\begin{array}{l}\text { Nadeem } \\
\text { et.al } \\
\text { (2014) }\end{array}$ & $\begin{array}{l}\text { Ibrahim et } \\
\text { al. (2017) }\end{array}$ & $\begin{array}{l}\text { Liao } \\
\text { (2011) }\end{array}$ & $\begin{array}{l}\text { Present } \\
\text { Study }\end{array}$ & $\begin{array}{l}\text { Nadeem } \\
\text { et.al } \\
\text { (2014) }\end{array}$ & $\begin{array}{l}\text { Ibrahim } \\
\text { et al. } \\
\text { (2017) }\end{array}$ & $\begin{array}{l}\text { Liao } \\
\text { (2011) }\end{array}$ & $\begin{array}{l}\text { Present } \\
\text { Study }\end{array}$ \\
\hline \multirow{2}{*}{0.2} & 0.3 & 0.4520 & 0.452019 & 0.4533 & 0.452083 & 0.8402 & 0.840133 & 0.8395 & 0.841270 \\
\hline & 0.5 & 0.3987 & 0.399937 & 0.3999 & 0.398601 & 0.8059 & 0.805741 & 0.8048 & $0 . .807752$ \\
\hline \multirow{2}{*}{3.0} & 0.3 & 0.4271 & 0.427223 & 0.4282 & 0.427081 & 0.7791 & 0.779103 & 0.7785 & 0.781528 \\
\hline & 0.5 & 0.3775 & 0.377607 & 0.3786 & 0.377137 & 0.7390 & 0.738916 & 0.8323 & 0.743303 \\
\hline \multirow{2}{*}{10} & 0.3 & 0.4216 & 0.421411 & 0.4277 & 0.421585 & 0.7660 & 0.766029 & 0.7654 & 0.768857 \\
\hline & 0.5 & 0.3728 & 0.373109 & 0.3739 & 0.372369 & 0.7248 & 0.724738 & 0.7238 & 0.729901 \\
\hline
\end{tabular}


Table 2: Numerical computations of skin friction coefficient $-\left(1+\frac{1}{\beta}\right) f^{\prime \prime}(0)$, local Nusselt number $-\left(1+\frac{4 R}{3}\right) \theta^{\prime}(0)$ and Sherwood number $-\phi^{\prime}(0)$ for various values of $H a, \beta, J, \alpha, Q, G r, G c$ when $n=2, \operatorname{Pr}=0.71, R=0.1, S=1.0, S c=0.6, N t=N b=0.1, E c=0.1$ and $\gamma=0.1$.

\begin{tabular}{|c|c|c|c|c|c|c|c|c|c|}
\hline $\mathrm{Ha}$ & $\beta$ & $\mathrm{J}$ & Q & $\alpha$ & $\mathrm{Gr}$ & $\mathrm{Gc}$ & $-\left(1+\frac{1}{\beta}\right) f^{\prime \prime}(0)$ & $-\left(1+\frac{4 R}{3}\right) \theta^{\prime}(0)$ & $-\phi^{\prime}(0)$ \\
\hline 0.1 & 1 & 0.5 & 0.1 & & 0.1 & 0.1 & 1.6678 & 0.2679 & 0.5338 \\
\hline 0.5 & & & & & & & 1.8958 & 0.2618 & 0.5218 \\
\hline 1 & & & & & & & 2.148 & 0.2531 & 0.5113 \\
\hline \multirow[t]{18}{*}{0.5} & 0.5 & & & & & & 2.3178 & 0.2738 & 0.5387 \\
\hline & 1 & & & & & & 1.8958 & 0.2618 & 0.5218 \\
\hline & 1.5 & & & & & & 1.7334 & 0.2527 & 0.5159 \\
\hline & & 0.5 & & & & & 2.3178 & 0.2738 & 0.5387 \\
\hline & & 1 & & & & & 2.3126 & 0.0781 & 0.6437 \\
\hline & & 1.5 & & & & & 2.3074 & -0.1188 & 0.7494 \\
\hline & & & -0.2 & & & & 2.3268 & 0.4997 & 0.4196 \\
\hline & & & $\mathbf{0}$ & & & & 2.3213 & 0.3576 & 0.4946 \\
\hline & & & 0.2 & & & & 2.3135 & 0.1775 & 0.5892 \\
\hline & & & & $\frac{\pi}{6}$ & & & 2.3178 & 0.2738 & 0.5387 \\
\hline & & & & $\frac{\pi}{4}$ & & & 2.3095 & 0.2739 & 0.5391 \\
\hline & & & & $\frac{\pi}{3}$ & & & 2.2988 & 0.2741 & 0.5397 \\
\hline & & & & & 0.1 & & 2.3178 & 0.2738 & 0.5387 \\
\hline & & & & & 0.3 & & 2.2105 & 0.2747 & 0.5441 \\
\hline & & & & & 0.5 & & 2.1048 & 0.2751 & 0.5495 \\
\hline & & & & & & 0.1 & 2.3178 & 0.2738 & 0.5387 \\
\hline & & & & & & 0.3 & 2.4087 & 0.2726 & 0.534 \\
\hline & & & & & & 0.5 & 2.5013 & 0.2709 & 0.5293 \\
\hline
\end{tabular}

\section{Conclusions}

The present study investigates the numerical solution of thermal radiation and Joule heating impact on magneto Casson Nanofluid with chemical reaction and heat source/sink on the inclined porous stretching surface. Keller Box method has successfully been implemented to carry out a relative analysis Under the impact of various physical conditions, the performance of dimensionless velocity, temperature, and concentration profiles are discussed. The important observations of this report are outlined and detailed below:

- Velocity and concentration profiles are diminishing with enhanced values of $\beta$.

- Velocity enhances with an increase of $\alpha$ but to reverse the temperature and concentration.

- An increasing value of Ec and $J$, the result in velocity and temperature increases.

- Temperature distribution enhances with an increase of Casson parameter $\beta$. 
- Velocity, Temperature and Concentration fields are decreases with the enhancing values of $S$.

- Increasing values of Hartmann number Ha the Velocity curves rises.

- Temperature increases when $H a, R, Q$, and $N t$ raises.

- With an increase of $N b$ and $\gamma$, the result in concentration is decreasing.

- With enhanced values of Ha and $\mathrm{n}$ the skin friction enhances as well as the reverse effect occurs with an increase of $S$ and $\beta$.

- Nusselt number rises with an increase of $G r$ and $R$ for changed values of $n$.

- Mass transfer rate increase with the changed values of $\gamma$ and $n$.

\section{Acknowledgements}

The authors are grateful and express sincere thanks to the reviewers for their detailed reading, precious proposals, and comments.

\section{References}

Adhikari, A., and Sanyal, D.C. (2013), Heat transfer on MHD viscous flow over a stretching sheet with prescribed heat flux, Bulletin of International Mathematical Virtual Institute, Vol. 3, pp.35-47.

Akbar, N.S., Tripathi, D., Anwar Bég, O., Khan, Z.H. (2016): MHD dissipative flow and heat transfer of casson fluids due to metachronal wave propulsion of beating cilia with thermal and velocity slip effects under an oblique magnetic field, Acta Astronautica, Vol. 128, pp. 1-12. https://doi.org/10.1016/j.actaastro.2016.06.044.

Akbar, N.S., Nadeem, S., Ul Haq, R., and Khan, Z.H. (2013): Radiation effects on MHD stagnation point flow of nano fluid towards a stretching surface with convective boundary condition, Chinese J. Aeronaut. Vol. 26, pp. 1389-1397. https://doi.org/10.1016/j.cja.2013.10.008.

Ali. M., Alim. M. A., Nasrin. R., and Alam. M. S. (2017): Numerical analysis of heat and mass transfer along a stretching wedge surface, Journal of Naval Architecture and Marine Engineering, Vol. 14, No. 2, pp. 135-144. http://dx.doi.org/10.3329/jname.v14i2.30633.

Arifuzzaman, S.M., Khan, M.S., Mehedi, M.F.U., Rana, B.M.J., and Ahmmed, S.F. (2018): Chemically reactive And naturally convective high speed MHD fluid flow through an oscillatory vertical porous plate with heat and Radiation absorption effect, Engineering Science and Technology, an International Journal, Vol. 21, No. 2, pp.215-228. https://doi.org/10.1016/j.jestch.2018.03.004.

Ashraf, M., and Bashir, S. (2011): Numerical simulation of MHD stagnation point flow and heat transfer of a micropolar fluid towards a heated shrinking sheet, International Journal of Numerical methods in fluids, Vol. 69, pp. 384-398. https://doi.org/https://doi.org/10.1002/fld.2564.

Babu, P.R., Rao, J.A., and Sheri, S. (2014): Radiation effect on MHD heat and mass transfer flow over a Shrinking sheet with mass suction, J. Appl. Fluid Mech., Vol. 7, pp.641-650. https://doi.org/10.36884/jafm.7.04.21389.

Bal Reddy, G., Shankar Goud, B., Raja Shekar, M.N. (2018): Keller box solution of magnetohydrodynamic boundary layer flow of nanofluid over an exponentially stretching permeable sheet, Int. J. Mech. Eng. Technol., Vol. 9(10), 2018, pp. 1646-1656.

Bala Anki Reddy. P (2016), MHD boundary layer slip flow of a Casson fluid over an exponentially stretching surface in the presence of thermal radiation and chemical reaction, Journal of Naval Architecture and Marine Engineering, Vol. 13, No. 2, pp. 165-177. http://dx.doi.org/10.3329/jname.v13i2.23537.

Banshiwal. A., and Goyal. M. (2018): MHD non-darcian flow due to horizontal stretching sheet embedded in a porous medium with thermal stratification effects, Journal of Naval Architecture and Marine Engineering, Vol.15, o. 1, pp. 65-73. http://dx.doi.org/10.3329/jname.v15i2.29692.

Bhattacharyya, K. (2013): MHD Stagnation-Point Flow of Casson Fluid and Heat Transfer, Vol. 2013, Article Id 169674, 9 pages. http://dx.doi.org/10.1155/2013/169674.

Choi, S U.S., and Eastman, J.A. (1995), Enhancing thermal conductivity of fluids with nanoparticles, J. Chem.Eng. Data., Vol. 12-17. https://www.osti.gov/servlets/purl/196525.

Daniel, Y.S., Aziz, Z.A., Ismail, Z., and Salah, F. (2018): Impact of thermal radiation on electrical MHD flow of nanofluid over nonlinear stretching sheet with variable thickness, Alexandria Eng. J. Vol. 57, pp. 2187-2197. https://doi.org/10.1016/j.aej.2017.07.007.

Dharmendar Reddy, Y., Ramya, D., and Babu, L.A. (2016): Effect of thermal radiation on MHD boundary layer flow of nanofluid and heat transfer over a non-linearly stretching sheet with transpiration, Journal of Nanofluids, Vol. 5, pp. 889-897. https://doi.org/10.1166/jon.2016.1284.

Dharmendar Reddy, Y., Rao, V.S., and Babu, L.A. (2017): Viscous dissipation and partial slip effects on MHD 
boundary layer flow of nanofluidand heat transfer over a nonlinear stretching sheet, International Journal of Mathematical Archive, Vol. 8, pp. 1-14.

Ferdows, M., Khan, M.S., Bég, O.A., Azad, M., and Alam, M.M. (2014): Numerical study of transient magnetohydrodynamic radiative free convection nanofluid flow from a stretching permeable surface, Proc. Inst. Mech. Eng. Part E J. Process Mech. Eng., Vol. 228,pp. 181-196. https://doi.org/10.1177/0954408913493406.

Geetha, P., Moorthy, M.B.K., and Dakshinamoorthy, M. (2014): Free convection of heat transfer in flow past a semi-infinite flat plate in transverse magnetic field with heat flux, Am. J. Appl. Sci. Vol. 11, pp. 1480-1485. https://doi.org/10.3844/ajassp.2014.1480.1485.

Hari Singh Naik, Shankar Goud, B., Suresh, P., and Ramana Murthy, M. V. (2020): Radiation and Hall Effect on MHD mixed convection of Casson fluid over a stretching sheet, Int. J. Adv. Sci. Technol., Vol. 29, No. 7, pp. 1121-1131.

Hayat, T., Shehzad, S.A., and Alsaedi, A.(2012): Soret and Dufour effects on magnetohydrodynamic (MHD) flowof Casson fluid, Appl. Math. Mech., Vol. 33, pp.1301-1312. https://doi.org/10.1007/s10483-012-1623-6. Ibrahim, S.M., Lorenzini, G., Vijaya Kumar, P., and Raju, C.S.K. (2017): Influence of chemical reaction and heat source on dissipative MHD mixed convection flow of a Casson nanofluid over a nonlinear permeable stretching sheet, Int. J. Heat Mass Transf. Vol. 111, pp. 346-355. https://doi.org/10.1016/j.ijheatmasstransfer.2017.03.097.

Liao, S. (2011): Homotopy analysis method in nonlinear differential equations, Homotopy Anal. Method Nonlinear Differ. Equations, ISBN No.978364225131, pp. 1-565. https://doi.org/10.1007/978-3-642-25132-0.

Khan, W.A., Makinde, O.D., and Khan, Z.H. (2014): MHD boundary layer flow of a nanofluid containing gyrotactic microorganisms past a vertical plate with Navier slip, International Journal of Heat and Mass Transfer, Vol. 74, pp. 285-291. https://doi.org/10.1016/j.ijheatmasstransfer.2014.03.026.

Khan, Z., Khan, I., Ullah, M., and Tlili, I. (2018): Effect of thermal radiation and chemical reaction on non Newtonian fluid through a vertically stretching porous plate with uniform suction, Results in Physics, Vol. 9, pp.1086-1095. https://doi.org/10.1016/j.rinp.2018.03.041.

Makinde, O.D., and Aziz, A. (2010): MHD mixed convection from a vertical plate embedded in a porous Medium with a convective boundary condition, Int. J. Therm. Sci., Vol. 49, pp.1813-1820. https://doi.org/10.1016/j.ijthermalsci.2010.05.015.

Makinde, O.D., and Aziz, A. (2011): Boundary layer flow of a nanofluid past a stretching sheet with a convective boundary condition, Int. J. Therm. Sci., Vol. 50, pp. 1326-1332. https://doi.org/10.1016/j.ijthermalsci.2011.02.019.

Mat Yasin, M.H., Ishak, A., and Pop, I. (2016): MHD heat and mass transfer flow over a permeable stretching/shrinking sheet with radiation effect, Journal of Magnetism and Magnetic Materials, Vol. 407, pp. 235-240. https://doi.org/10.1016/j.jmmm.2016.01.087.

Mohamed, R.A., and Abo-Dahab, S.M. (2009), Influence of chemical reaction and thermal radiation on the heat and mass transfer in MHD micropolar flow over a vertical moving porous plate in a porous medium with heat generation, Int. J. Therm. Sci., Vol. 48, pp. 1800-1813. https://doi.org/10.1016/j.ijthermalsci.2009.01.019.

Mukhopadhyay, S., De, P.R., Bhattacharyya, K., and Layek, G.C. (2013): Casson fluid flow over an unsteady stretching surface, Ain Shams Engineering Journal, Vol. 4, No. 4, pp. 933-938. https://doi.org/10.1016/j.asej.2013.04.004.

Mustafa, M., Hayat, T., Pop, I., and Aziz, A. (2011): Unsteady boundary layer flow of a Casson fluid due to an impulsively started moving flat plate, Heat Transf.-Asian Res., Vol. 40, pp.563-576. https://doi.org/10.1002/htj.20358.

Nadeem, S., Haq, R.U., and Akbar, N.S. (2014): MHD three-dimensional boundary layer flow of casson nanofluid past a linearly stretching sheet with convective boundary condition, IEEE Trans. Nanotechnol. Vol. 13, pp. 109-115. https://doi.org/10.1109/TNANO.2013.2293735.

Nandy, S.K. (2013): Analytical Solution of MHD Stagnation-Point Flow and Heat Transfer of Casson Fluid over a Stretching Sheet with Partial Slip, ISRN Thermodynamics., Vol. 2013, pp. 1-9. https://doi.org/10.1155/2013/108264.

Nandeppanavar, M.M., Vajravelu, K., and Subhas Abel, M. (2011): Heat transfer in MHD viscoelastic boundary layer flow over a stretching sheet with thermal radiation and non-uniform heat source/sink, Commun. Nonlinear Sci. Numer. Simul., Vol. 16,pp. 3578-3590. https://doi.org/10.1016/j.cnsns.2010.12.033.

Nayak, M.K., Dash, and G.C., Singh, L.P. (2016): Heat, and mass transfer effects on MHD viscoelastic fluid over a stretching sheet through a porous medium in presence of chemical reaction, Propulsion and Power Research, Vol. 5, No. 1, pp. 70-80. https://doi.org/10.1016/j.jppr.2016.01.006.

Nayak, M.K., Akbar, N.S., Pandey, V.S., Khan, Z.H., and Tripathi, D. (2017): 3D free convective MHD flow of nanofluid over permeable linear stretching sheet with thermal radiation, Powder Technology. Vol. 315, pp. 2015-215. https://doi.org/10.1016/j.powtec.2017.04.017.

Thermal radiation and joule heating effects on a magnetohydrodynamic Casson Nanofluid flow in the presence of chemical ... 
Palani, G., and Kim, K.Y. (2012), Influence of magnetic field and thermal radiation by natural convection past vertical cone subjected to variable surface heat flux, Appl. Math. Mech., Vol. 33, pp.605-620. https://doi.org/10.1007/s10483-012-1574-7.

Pekmen Geridonmez, B., and Oztop, H.F. (2019): Natural convection in a cavity filled with porous medium under the effect of a partial magnetic field, International Journal of Mechanical Sciences, Vol. 161/162. https://doi.org/10.1016/j.ijmecsci.2019.105077.

Rajput. U. S., and Gaurav Kumar (2019): Effects of radiation and chemical reaction on MHD flow past a vertical plate with variable temperature and mass diffusion, Journal of Naval Architecture and Marine Engineering, Vol. 16, No. 2, pp. 99-108. http://dx.doi.org/10.3329/jname.v16i2.29526.

Raptis, A.A., Kafousias, N.G., and Massalas, C. V. (1982): Free Convection and Mass Transfer Flow through a Porous Medium Bounded by an Infinite Vertical Porous Plate with Constant Heat Flux, ZAMM - Journal of Applied Mathematics and Mechanics, Vol. 62, No. 9, pp. 489-491. https://doi.org/10.1002/zamm.19820620911. Rizwan-Ul-Haq., Nadeem, S., Khan, Z.H. and Okedayo, T.G. (2014): Convective heat transfer and MHD effects on Casson nanofluid flow over a shrinking sheet, Vol. 12, No. 12, pp. 862-871. https://doi.org/10.2478/s11534014-0522-3.

Shankar Goud, B., and Rajashekar, M.N. (2018): Implicit finite difference method for mhd flow of a micropolar fluid past a stretching sheet with heat transfer, fifth Int. Conf. Comput. Methods Therm. Probl., 2018, July 9-11, 2018, Indian Institute of Science, Bangalore, INDIA.

Shankar Goud, B. (2020): Thermal Radiation Influences on MHD Stagnation Point Stream over a Stretching Sheet with Slip Boundary Conditions, International Journal of Thermofluid Science and Technology, Vol. 7, pp. 1-11. https://doi.org/10.36963/IJTST.2020070201.

Shankar Goud, B. (2020): Heat generation / absorption influence on steady stretched permeable surface on MHD flow of a micropolar fluid through a porous medium in the presence of variable suction / injection, Int. J. Thermofluids, Vol. 7-8, 100044. https://doi.org/10.1016/j.ijft.2020.100044.

Sharma. P., and Saboo. R. (2017): Heat and mass transfer with viscous dissipation in horizontal channel partially occupied by porous medium in the presence of oscillatory suction, Journal of Naval Architecture and Marine Engineering, Vol. 14, No. 2, pp. 101-114. http://dx.doi.org/10.3329/jname.v14i2.25584

Shima, P.D., Philip, J., and Raj, B. (2009): Magnetically controllable nanofluid with tunable thermal conductivity and viscosity, Appl. Phys. Lett., Vol. 95,pp.16-19. https://doi.org/10.1063/1.3238551.

Sui, J., Zheng, L., Zhang, and Chen, G. (2015): Mixed convection heat transfer in power law fluids over a moving conveyor along an inclined plate, Int. J. Heat Mass Transf., Vol. 85, pp.1023-1033. https://doi.org/10.1016/j.ijheatmasstransfer.2015.02.014. 